\title{
Characteristics-based boundary conditions for the Euler adjoint problem
}

\author{
Marcelo Hayashi ${ }^{1, *, \dagger}$, Marco Ceze $^{2}$ and Ernani Volpe ${ }^{1}$ \\ ${ }^{1}$ University of São Paulo, São Paulo, 05508-970, Brazil \\ ${ }^{2}$ University of Michigan, Ann Arbor, MI, 48105, USA
}

\begin{abstract}
SUMMARY
Over the last decade, the adjoint method has been consolidated as one of the most versatile and successful tools for aerodynamic design. It has become a research area on its own, spawning a large variety of applications and a prolific literature. Yet, some relevant aspects of the method remain relatively less explored in the literature. Such is the case with the adjoint boundary problem. In particular for Euler flows, both fluid dynamic and adjoint equations entail complementary Riemann problems, and these yield boundary conditions that are fully consistent with well-posedness. In the literature, this approach has been pursued solely in terms of Riemann variables. This work formulates the adjoint boundary problem so as to correspond precisely to that imposed on the flow, as it is given in terms of primitive variables. Test results have shown to be in agreement with the traditional approach for external flow problems. Copyright $\odot 2012$ John Wiley \& Sons, Ltd.
\end{abstract}

Received 6 March 2012; Revised 15 June 2012; Accepted 23 June 2012

KEY WORDS: adjoint method; aerodynamic design; boundary conditions; Euler flow

\section{INTRODUCTION}

The problem of inverse aerodynamic design was first considered by Lighthill, in 1945, and the investigation was limited to airfoils in incompressible potential flows [1]. The interest in the topic has grown ever since, following closely on the progresses in computational resources and numerical methods for flow simulation. Over the years, a variety of methods have been proposed to tackle the problems of aerodynamic optimization and inverse design.

The adjoint method has played a prominent role in that context, for a number of reasons. Among them, one could cite the great flexibility it offers with regard to the flow-physics model and to the definition of objective functionals. Originally proposed by Pirronneau [2-4] for elliptic problems, it was later extended to transonic flows by Jameson [1]. Since then, it has become the subject of extensive research activity [5-14] and spawned a wide variety of applications, ranging from nuclear reactor thermo-hydraulics to atmospheric sciences $[15,16]$. In aerodynamics, the developments of the adjoint method encompass design applications regarding internal and external flows [17-22] and, more recently, unsteady flows [23-26].

To put matters into perspective, objective functionals of general interest in aerodynamics depend on flow variables and on the shape and location of the boundaries [27, 28]. These, in turn, are controlled by a set of design parameters. For all practical purposes, the set is assumed to be finite. Under these circumstances, a natural means of estimating the sensitivity of that functional to changes in flow geometry would be to perturb each design parameter individually and then to compute the sensitivity gradient by finite differences.

*Correspondence to: Marcelo Hayashi, Av. Prof. Mello Moraes, 2231, São Paulo, 05508-970, Brazil.

†E-mail: mhayashi@usp.br 
The procedure clearly requires a converged flow solution for each parameter variation. As the number of design parameters increases, so does the number of solutions, and the computational cost is bound to become prohibitive. Alternatively, by imposing the equations that govern the flow as constraints to the optimization problem, one precludes unrealizable solutions. That, in turn, eliminates the need for additional flow simulations. Therein lies the essence of the adjoint method. As a result of the simplification, the method offers an extremely attractive capability, which is to compute sensitivity gradients at a cost that is largely independent of the number of design parameters.

The formulation of the adjoint problem has evolved into two distinct approaches: discrete and continuous. In the former, a discrete adjoint operator is obtained on the basis of already discretized flow governing equations. Whereas in the latter case, the adjoint equations are derived analytically from the equations that govern the flow. Comparisons between the two approaches have also been drawn in the literature [28-30], and they do not seem to indicate any sizable differences between them in terms of optimization effectiveness.

On the other hand, the continuous formulation offers a clear insight into the nature of the adjoint problem [31]. To gain access to it, Giles and Pierce [31-34] have conducted a thorough investigation of the mathematical properties of adjoint equations in the continuous form. Of particular interest to our purposes, in reference [31], the authors explore the hyperbolic character of the Euler and their adjoint equations to ensure both problems are well posed. They cast them in terms of Riemann variables, and make use of the fact that flow and adjoint characteristic velocities have opposite signs. That gives the boundary problems a complementary character, thus paving the way to make the adjoint equations well posed.

Yet, there is one aspect of the procedure that deserves further consideration: the results have been entirely derived in terms of Riemann variables. Although that does suit the main purpose of proving the flow and adjoint boundary problems are complementary, it does not correspond directly to the way boundary conditions are usually specified, for that is most often carried out in terms of primitive variables instead. To obtain these from the original expressions, it would require the corresponding Riemann variables to be integrated, and that cannot be carried out in closed form for all cases of interest [35].

This work proposes a means of circumventing the difficulty. It makes use of the fact that the boundary problems are complementary and of Riemann equations as well. But it combines them with the idea that the bilinear concomitant the adjoint problem entails is an inner product between physical variations and adjoint variables [15]. The product is computed over the domain boundary, where any physical variation is governed by flow boundary conditions.

In essence, boundary conditions determine which physical variations are realizable. Then, the need to drive the bilinear concomitant to zero is seen as an orthogonality condition. That is, the adjoint variables define a vector in state space, which must be orthogonal to any realizable variation of the flow physics. The rationale is analogous to that behind the principle of virtual work, in that the adjoint vector plays the part of a generalized constraint force, which should be normal to any acceptable virtual displacement of the system. The approach is shown to be fully consistent with the requirements for well-posedness of the adjoint equation, as they are determined by the Riemann problem [36-38].

A comparison between this approach and that by Giles and Pierce [31] is drawn in the following section in terms of the resultant equations. It shows that although the former yields a bit more complex algebraic expressions, it does share with the latter in the linearity of the adjoint boundary conditions, as it is expected to. Test results validate the proposed approach in applications of inverse aerodynamic design.

\section{THE VARIATIONAL PROBLEM}

As was mentioned earlier, the objective functionals of interest in aerodynamics depend on the flow variables and on the geometry of the boundaries. In principle, these functionals could either be volume integrals over the flow domain or surface integrals over a boundary such as the body surface, for instance. The latter case is of special relevance to us, for it includes a variety of aerodynamic 
measures of merit, ranging from force coefficients to inverse design. A fairly general representation of these functionals would be

$$
I_{o}=\int_{B_{w}} g(\mathbf{V}) \mathrm{d} S
$$

where $B_{w}$ stands for the body surface, a function of space coordinates that is fully specified by a finite set of parameters $B_{w} \Rightarrow S\left(\xi^{j} ; a^{k}\right)$. The quantity $g(\mathbf{V})$ represents a scalar function of the flow variables $\mathbf{V}=\left(\rho, u^{i}, p\right)^{T}$ — which can naturally be put in terms of the conservative variables $\mathbf{Q}=\left(\rho, \rho u^{i}, e\right)^{T}$ as well.

A relevant problem would be to find out a surface geometry that leads to a minimum of $I_{o}$ under given flow conditions. In the framework of the adjoint method, it implies evaluating the sensitivity of $I_{o}$ to changes in the control parameters $a_{k}$. To first order, that can be estimated by the sensitivity gradient $\partial I_{o} / \partial a^{k}$.

Parameter variations clearly cause the body surface to change, thereby perturbing the flow field as well, $\delta a^{k} \Rightarrow \delta S \Rightarrow \delta \mathbf{Q}$. A convenient means of separating physical from geometric variations is to define a spatial transformation, whereby the body surface is mapped onto a constant coordinate plane. It also simplifies the variational problem considerably, in that the domains of integration remain fixed in transformed space [39]. All that change in the process are the transformation operators and the metrics of the latter.

To that end, we take the physical space to be represented by Cartesian coordinates $x^{i^{\prime}}$, and the transformed space by generalized coordinates $\xi^{k}$ - to distinguish between the two systems, all tensor elements in Cartesian coordinates will hereafter be designated by primed indices, and unprimed indices are reserved for generalized elements. The transformation operator and its inverse are defined by, respectively,

$$
\beta \Rightarrow \beta_{j}^{i^{\prime}}=\frac{\partial x^{i^{\prime}}}{\partial \xi^{j}} \quad ; \quad \beta^{-1} \Rightarrow \beta_{i^{\prime}}^{j}=\frac{\partial \xi^{j}}{\partial x^{i^{\prime}}}
$$

The transformation Jacobian is $J=\operatorname{det}\left(\beta_{j}^{i^{\prime}}\right)$, and it is assumed to be independent of time. It is also understood that $J$ should not go to zero nor change its sign anywhere in $\mathcal{D}$. In this context, the first variation of the objective functional (1) is given by

$$
\delta I_{o}=\int_{\boldsymbol{B}_{w}} \frac{\partial g}{\partial \mathbf{V}} \frac{\partial \mathbf{V}}{\partial \mathbf{Q}} \delta \mathbf{Q}\left|\frac{\mathrm{d} S^{\prime}}{\mathrm{d} S}\right| \mathrm{d} S+\int_{\boldsymbol{B}_{w}} g \delta\left|\frac{\mathrm{d} S^{\prime}}{\mathrm{d} S}\right| \mathrm{d} S
$$

where $\mathrm{d} S^{\prime}$ and $\mathrm{d} S$ represent area elements in Cartesian and generalized coordinates, respectively. Equation(3) naturally involves physical and geometric variations. The latter term in it can usually be derived from $S\left(\xi^{i} ; a_{k}\right)$ analytically, whereas the former is hardly ever known in closed form. In effect, it is the presence of that term in $\delta I_{o}$ that gives rise to the need for additional flow simulations.

In order to circumvent such need, one imposes the fluid mechanics equations as constraints on the variational problem. They play the essential role of realizability conditions, which all physical variations $\delta \mathbf{Q}$ must satisfy. In the present case, we pick the Euler equations, which are given in Cartesian coordinates by

$$
\frac{\partial Q_{\alpha}}{\partial t}+\frac{\partial f_{\alpha}^{k^{\prime}}}{\partial x^{k^{\prime}}}=0
$$

where Greek subscripts range from 1 to 5, indicating coordinates in state space: 1 refers to continuity, 2-4 to linear momentum and 5 to the energy equation. The state $Q_{\alpha}$ and flux $f_{\alpha}^{k^{\prime}}$ vectors are defined as

$$
Q_{\alpha} \Rightarrow\left(\frac{\rho}{\rho u^{i^{\prime}}} \frac{;}{e}\right) \quad f_{\alpha}^{k^{\prime}} \Rightarrow\left(\frac{\rho u^{k^{\prime}}}{\frac{\rho u^{i^{\prime}} u^{k^{\prime}}+p g^{i^{\prime} k^{\prime}}}{(e+p) u^{k^{\prime}}}}\right)
$$


In particular for the momentum equations, there is a relation between the superscript $i^{\prime}$ and the subscript $\alpha: i^{\prime}=\alpha-1$ for $2 \leqslant \alpha \leqslant 4$. The symbol $e$ represents total energy, $e=\rho\left(e_{i}+\mathbf{u} \cdot \mathbf{u} / 2\right), e_{i}$ denotes the specific internal energy and the $g^{i^{\prime} j^{\prime}}$ stands for the metric tensor, which is the identity matrix in Cartesian coordinates. The set is closed by the ideal gas relation between pressure and internal energy

$$
p=\rho e_{i}(\gamma-1)
$$

The Euler equations in transformed space are obtained by a simple application of the $\beta$ operators [40]:

$$
\begin{array}{r}
J \frac{\partial Q_{\alpha}}{\partial t}+J \beta_{i^{\prime}}^{k} \frac{\partial f_{\alpha}^{i^{\prime}}}{\partial \xi^{k}}=0 \\
\frac{\partial\left(J Q_{\alpha}\right)}{\partial t}+\frac{\partial F_{\alpha}^{k}}{\partial \xi^{k}}=0
\end{array}
$$

A tensorial identity [41] is crucial to derive their latter form, and it is also implied in the definition of generalized flux vectors $F_{\alpha}^{k}$,

$$
\frac{\partial\left(J \beta_{i^{\prime}}^{k}\right)}{\partial \xi^{k}}=0 \quad \Rightarrow \quad F_{\alpha}^{k}=J \beta_{i^{\prime}}^{k} f_{\alpha}^{i^{\prime}}
$$

Generalized flux-Jacobian matrices are then defined on the basis of Equation (8)

$$
C_{\alpha \beta}^{k}=J \beta_{i^{\prime}}^{k} \frac{\partial f_{\alpha}^{i^{\prime}}}{\partial Q_{\beta}}=J \beta_{i^{\prime}}^{k} A_{\alpha \beta}^{i^{\prime}}
$$

and they lead to the following form of the generalized Euler equations

$$
\frac{\partial Q_{\alpha}}{\partial t}+\frac{C_{\alpha \beta}^{k}}{J} \frac{\partial Q_{\beta}}{\partial \xi^{k}}=0
$$

Either in this form, or as it appears in (7), the Euler equations can be imposed on the variational problem as realizability constraints.

In the applications that are considered here, one is mostly interested in steady flow conditions. Therefore, the steady form of (7) is imposed on (1) as a non-holonomic constraint in transformed space. The procedure leads to an augmented functional, which is given by

$$
I=\underbrace{\int_{B_{w}} g(\mathbf{V})\left|\frac{\mathrm{d} S^{\prime}}{\mathrm{d} S}\right| \mathrm{d} S}_{I_{o}}+\underbrace{\int_{\mathcal{D}} \Psi_{\alpha} \frac{\partial F_{\alpha}^{k}}{\partial \xi^{k}} \mathrm{~d} \mathcal{V}}_{I_{C}}
$$

Here, the $I_{c}$ indicates the constraint functional, which is a volume integral over the flow domain, $\mathcal{D}$, as a whole. It is understood that the ratio between Cartesian and generalized volume elements is given by transformation Jacobian itself, $\mathrm{d} \mathcal{V}^{\prime} / \mathrm{d} \mathcal{V}=J$. The vector $\Psi_{\alpha}$ represents Lagrange multipliers, and it is of the same dimension as the state vector $Q_{\alpha}$.

The first variation of $I_{o}$ is already given in (3), whereas that of $I_{c}$ must still be computed. It involves the variation of the constraint equation, which is given by

$$
\frac{\partial}{\partial \xi^{k}}\left(\delta F_{\alpha}^{k}\right)=0
$$

The generalized flux variation is obtained by combining Equations (8) and (9), and the procedure yields

$$
\begin{aligned}
\delta F_{\alpha}^{k} & =J \beta_{i^{\prime}}^{k} A_{\alpha \beta}^{i^{\prime}} \delta Q_{\beta}+\delta\left(J \beta_{i^{\prime}}^{k}\right) f_{\alpha}^{i^{\prime}} \\
& =C_{\alpha \beta}^{k} \delta Q_{\beta}+\delta\left(J \beta_{i^{\prime}}^{k}\right) f_{\alpha}^{i^{\prime}}
\end{aligned}
$$


It is worth noting that the first term on the right-hand side (RHS) of (13) can be regarded as the physical part of the flux variation, whereas the second one represents the geometric part.

The first variation of $I_{c}$ is a direct result of Equation (12), and it can be further simplified by making use of Gauss' theorem.

$$
\begin{aligned}
\delta I_{c} & =\int_{\mathcal{D}} \Psi_{\alpha} \frac{\partial}{\partial \xi^{k}}\left(\delta F_{\alpha}^{k}\right) \mathrm{d} \mathcal{V} \\
& =\int_{\partial \mathcal{D}} \Psi_{\alpha} \delta F_{\alpha}^{k} n_{k} \mathrm{~d} S-\int_{\mathcal{D}} \delta F_{\alpha}^{k} \frac{1}{J} \frac{\partial\left(J \Psi_{\alpha}\right)}{\partial \xi^{k}} \mathrm{~d} \mathcal{V}
\end{aligned}
$$

where the symbol $\partial \mathcal{D}$ indicates the whole boundary of the flow domain, which includes the body surface $\left(B_{w}\right)$, the far field $\left(B_{\infty}\right)$ and cut planes $\left(B_{c p}\right)$. In effect, the surface integral in Equation (14) defines the bilinear concomitant of the problem in its most general form. The term $n_{k}$ represents a normal unit vector that points outward from the flow domain.

Naturally, the variation of the augmented functional (11) is given by the sum of Equations (3) and (14): $\delta I=\delta I_{o}+\delta I_{c}$. On adding them up and on separating the surface integrals according to each boundary, one obtains

$$
\begin{aligned}
\delta I= & \int_{B_{w}} \frac{\partial g}{\partial Q} \delta Q\left|\frac{\mathrm{d} S^{\prime}}{\mathrm{d} S}\right| \mathrm{d} S+\int_{B_{w}} g \delta\left|\frac{\mathrm{d} S^{\prime}}{\mathrm{d} S}\right| \mathrm{d} S-\int_{\mathcal{D}} \delta F_{\alpha}^{k} \frac{1}{J} \frac{\partial\left(J \Psi_{\alpha}\right)}{\partial \xi k} \mathrm{~d} \mathcal{V}+ \\
& +\int_{B_{w}} \Psi_{\alpha} \delta F_{\alpha}^{k} n_{k} \mathrm{~d} S+\int_{B_{\infty}} \Psi_{\alpha} \delta F_{\alpha}^{k} n_{k} \mathrm{~d} S+\left[\int_{B_{c p}} \Psi_{\alpha} \delta F_{\alpha}^{k} n_{k} \mathrm{~d} S\right]_{c p^{+}}^{c p^{-}}
\end{aligned}
$$

Here, the square brackets imply that the flux variations are integrated over both sides of each cut plane $B_{c p}$. Owing to the fact that the normal has opposite orientation on each side, any cut plane yields a difference between two similar integrals, one over each side. Moreover, the fluxes should be continuous through the cut planes to be physically realizable, and the same must hold for their variations. Under these circumstances, one can eliminate that term from Equation (15) by simply imposing periodic boundary conditions on the adjoint variables at the cut planes,

$$
\left.\Psi_{\alpha}\right|_{c p^{+}}=\left.\Psi_{\alpha}\right|_{c p^{-}}
$$

It requires that the $\Psi_{\alpha}$ and all of their derivatives be continuous through $B_{c p}$.

Another condition for physical realizability implies that the normal velocity at the solid walls should be zero, $\mathbf{u} \cdot \mathbf{n}=0$. That requirement constrains the flux vector normal to $B_{w}$, and it should also hold for its first variation.

$$
\left.F_{\alpha}^{k} n_{k}\right|_{B_{w}}=\left.\left(\begin{array}{c}
0 \\
p J \beta_{i^{\prime}}^{k} g^{i^{\prime} j^{\prime}} n_{k} \\
0
\end{array}\right) \Rightarrow \delta F_{\alpha}^{k} n_{k}\right|_{B_{w}}=\left(\begin{array}{c}
0 \\
\delta p J \beta_{i^{\prime}}^{k} i^{i^{\prime} j^{\prime}} n_{k} \\
0
\end{array}\right)+\left(\begin{array}{c}
0 \\
p \delta\left(J \beta_{i^{\prime}}^{k}\right) g^{i^{\prime} j^{\prime}} n_{k} \\
0
\end{array}\right)
$$

and on assuming that wall is mapped onto a constant coordinate plane, say $B_{w} \Rightarrow \xi^{2}=0$, one can write

$$
\left.\Psi_{\alpha} \delta F_{\alpha}^{k} n_{k}\right|_{\xi^{2}=0}=\delta p\left[\Psi_{\left(i^{\prime}+1\right)} J \beta_{i^{\prime}}^{2} n_{2}\right]+p\left[\Psi_{\left(i^{\prime}+1\right)} \delta\left(J \beta_{i^{\prime}}^{2}\right) n_{2}\right]
$$

where use was made of the relation $\alpha=i^{\prime}+1$, which holds for the momentum equations and was mentioned in regard to Equation (5). It is also implied that the metric tensor coincides with the identity matrix in the Cartesian system. 
On substituting Equations (16) and (17) for the corresponding terms in Equation (15), and on replacing the flux variation by Equation (13), one obtains the final expression for $\delta I$

$$
\begin{aligned}
& \delta I=\underbrace{\int_{B_{w}}\left\{\frac{\partial g}{\partial Q_{\beta}}\left|\frac{\mathrm{d} S^{\prime}}{\mathrm{d} S}\right|+\left[\Psi_{\left(i^{\prime}+1\right)} J \beta_{i^{\prime}}^{2} n_{2}\right] \frac{\partial p}{\partial Q_{\beta}}\right\} \delta Q_{\beta} \mathrm{d} S}_{(\mathrm{a})}+\underbrace{\int_{B_{\infty}} \Psi_{\alpha} C_{\alpha \beta}^{2} \delta Q_{\beta} n_{2} \mathrm{~d} S}_{(\mathrm{b})}+ \\
& -\underbrace{\int_{\mathcal{D}} \frac{C_{\alpha \beta}^{k}}{J} \frac{\partial\left(J \Psi_{\alpha}\right)}{\partial \xi^{k}} \delta Q_{\beta} \mathrm{d} \mathcal{V}}_{(\mathrm{c})}+\underbrace{\int_{B_{w}}\left\{g \delta\left|\frac{\mathrm{d} S^{\prime}}{\mathrm{d} S}\right|+p\left[\Psi_{\left(i^{\prime}+1\right)} \delta\left(J \beta_{i^{\prime}}^{2}\right) n_{2}\right]\right\} \mathrm{d} S}_{(\mathrm{d})}+ \\
& +\underbrace{\int_{B_{\infty}} \Psi_{\alpha} \delta\left(J \beta_{i^{\prime}}^{2}\right) f_{\alpha}^{i^{\prime}} n_{2} \mathrm{~d} S}_{(\mathrm{e})}-\underbrace{\int_{\mathcal{D}}^{\frac{\delta\left(J \beta_{i^{\prime}}^{k}\right) f_{\alpha}^{i^{\prime}}}{J} \frac{\left(J \Psi_{\alpha}\right)}{\partial \xi^{k}} \mathrm{~d} \mathcal{V}}}_{(\mathrm{f})}
\end{aligned}
$$

Here, it has been assumed that the far-field boundary is also mapped onto a constant coordinate plane, $B_{\infty} \Rightarrow \xi^{2}=1$. A separation between physical and geometric variations is easily noticed in Equation (18). The first three terms, (a), (b) and (c), belong in the former group. They give rise to the adjoint problem, which is constructed with the specific purpose of eliminating them from the total variation, $\delta I$. The remaining three integrals, (d), (e) and (f), involve only geometric variations, and they can be carried over into the sensitivity gradient as they are [36]. However, Jameson and Sangho [41] have proposed a further simplification to the gradient expression. They have shown that one can use the reduced form

$$
\begin{aligned}
& \delta I=\underbrace{\int_{B_{w}}\left\{\frac{\partial g}{\partial Q_{\beta}}\left|\frac{\mathrm{d} S^{\prime}}{\mathrm{d} S}\right|-\left[\Psi_{\left(i^{\prime}+1\right)} J \beta_{i^{\prime}}^{2} n_{2}\right] \frac{\partial p}{\partial Q_{\beta}}\right\} \delta Q_{\beta} \mathrm{d} S}_{\text {(a) }}+\underbrace{\int_{B_{\infty}} \Psi_{\alpha} C_{\alpha \beta}^{2}\left(\delta Q_{\beta}-\delta Q_{\beta}^{*}\right) n_{2} \mathrm{~d} S+}_{\text {(b) }} \\
& -\underbrace{\int_{\mathcal{D}} \frac{C_{\alpha \beta}^{k}}{J} \frac{\partial\left(J \Psi_{\alpha}\right)}{\partial \xi^{k}}\left(\delta Q_{\beta}-\delta Q_{\beta}^{*}\right) \mathrm{d} \mathcal{V}}_{\text {(c) }}+\underbrace{\int_{B_{w}} \Psi_{\alpha}\left[\delta\left(J \beta_{i^{\prime}}^{2}\right) f_{\alpha}^{i^{\prime}}+C_{\alpha \beta}^{2} \delta Q_{\beta}^{*}\right] n_{2} \mathrm{~d} S}_{\text {1st grad. term }}+ \\
& +\underbrace{\int_{B_{w}}\left\{g \delta\left|\frac{\mathrm{d} S^{\prime}}{\mathrm{d} S}\right|+p\left[\Psi_{\left(i^{\prime}+1\right)} \delta\left(J \beta_{i^{\prime}}^{2}\right) n_{2}\right]\right\} \mathrm{d} S}_{\text {2nd grad. term }}
\end{aligned}
$$

Quite different from (18), in Equation (19), the sensitivity gradient amounts to just two integrals over the body surface $B_{w}$. The gradient integrals over $\mathcal{D}$ and $B_{\infty}$ from the former equation have been removed, but an additional variation term, $\delta Q_{\beta}^{*}$, has appeared in their stead. It refers to variations in the state variables that are owed to mesh movement $\delta \xi^{k}$ alone, at a fixed boundary configuration [41]. Despite their particular cause, $\delta \xi^{k}$, they are of the same nature as the original $\delta Q_{\beta}$, in that they must also satisfy the Euler equations. For simplicity, the symbol $\delta \bar{Q}_{\beta}$ will be used to denote the difference: $\delta \bar{Q}_{\beta}=\delta Q_{\beta}-\delta Q_{\beta}^{*}$.

\section{THE ADJOINT EULER EQUATION}

The goal of removing all physical variations from the expression of $\delta I$ implies that the first three operators in Equation (19) should vanish. The conditions under which that can be accomplished define the adjoint problem, as was mentioned previously. 
For that purpose, term (c) can be regarded as an inner product between $\delta \bar{Q}_{\beta}$ and a differential operator, which is applied to $\Psi_{\alpha}$. Because all physical variations must be realizable, but they are otherwise arbitrary, it follows that the only means of eliminating the product is to require that the term involving $\Psi_{\alpha}$ be identically zero over $\mathcal{D}$ as a whole. The procedure gives rise to the adjoint equation in steady form.

It is not difficult to notice a similarity between the differential operator in term (c), Equation (19), and the steady form of the Euler equations (10). In principle, that enables one to formulate the adjoint problem in a way that is similar to the flow problem.

It is well known that the Euler equations hyperbolic character is what ultimately determines their boundary problem. It also sets the conditions for their being well posed. On having the adjoint equation share in the same character, one may extend the similarity to the adjoint boundary problem as well. That can be accomplished by simply postulating that $\Psi_{\alpha}$ depends on time in a way that is similar to $Q_{\beta}$. This step leads to the following adjoint partial differential equations (PDEs):

$$
\begin{aligned}
\frac{\partial \Psi_{\alpha}}{\partial t}-\frac{C_{\beta \alpha}^{k}}{J} \frac{\partial\left(J \Psi_{\beta}\right)}{\partial \xi^{k}} & =0 \\
\frac{\partial Q_{\alpha}}{\partial t}+\frac{C_{\alpha \beta}^{k}}{J} \frac{\partial Q_{\beta}}{\partial \xi^{k}} & =0
\end{aligned}
$$

which are paired with the flow governing equations (21) for comparison. It is worth noting here that the flux Jacobian matrix is transposed in the adjoint equations (20). One may also add that, despite their appearance, the adjoint equations are not in divergence form. For simplicity, then, one can redefine the adjoint variable so as to take in the transformation Jacobian, $\psi_{\alpha} \equiv J \Psi_{\alpha}$. It lends the adjoint equations the following form:

$$
\begin{aligned}
\frac{\partial \psi_{\alpha}}{\partial t}-C_{\beta \alpha}^{k} \frac{\partial \psi_{\beta}}{\partial \xi^{k}} & =0 \\
\frac{\partial \boldsymbol{\psi}}{\partial t}-\left(\mathbf{C}^{T} \cdot \nabla\right) \boldsymbol{\psi} & =0
\end{aligned}
$$

where the symbolic notation has been introduced for future convenience.

Clearly, one is only interested in the steady-state solution to Equation (22), for that solution alone drives term (c) in Equation (19) to zero. The point in casting the adjoint PDEs in this form is that not only do they share in the hyperbolic character of the Euler equations, but they also have the same characteristics. Their major difference lies in the sign reversal of the adjoint characteristic velocities. Quite distinct from the Euler equations, although, the adjoint PDEs are linear, because the flux Jacobian matrices do not depend on $\psi_{\alpha}$.

Both features, linearity and hyperbolicity, form the basis of the adjoint boundary problem. Nonetheless, the boundary conditions should fulfill two requirements: they must rid the sensitivity gradient (19) of physical variations and they must also be consistent with the well-posedness of the adjoint equations (22). As will be shown next, the sign reversal of the adjoint characteristics plays a crucial role in ensuring that the aforementioned requirements are met.

\subsection{Adjoint boundary conditions}

At least in part, the adjoint boundary problem was approached in Section 2 with regard to the cut planes. The need to drive the last term in Equation (15) to zero, by itself, would only require that $\psi_{\alpha}$ be continuous through the cut plane. Yet, to be consistent with the well-posedness of the adjoint PDEs, one must impose continuity up to first order derivatives-given that the cut plane should not coincide with any characteristic surface [42]—hence, the reason for imposing periodic boundary conditions through Equation (16).

With respect to solid walls, Equation (17) represents a realizability constraint. The fact that the wall is impermeable implies that the normal velocity and its variation should both be zero. When it is introduced in the expression for $\delta I,(18)-(19)$, it gives rise to an integral (a) over $B_{w}$, which is also related to the objective functional, through the derivative $\partial g / \partial Q_{\beta}$. 
The need to drive that integral to zero, while accounting for the fact that $\delta Q_{\beta}$ is arbitrary (albeit realizable), leads to a single wall boundary condition. It can be cast in an alternative form that suits best our purposes by making use of the chain rule in state space [36] and by substituting $\psi_{\alpha}$ for the corresponding product.

$$
\Psi_{\left(i^{\prime}+1\right)} J \beta_{i^{\prime}}^{2} n_{2}=\left.\psi_{\left(i^{\prime}+1\right)} n_{i^{\prime}}\right|_{B_{w}}=\frac{\partial g}{\partial p}\left|\frac{\mathrm{d} S^{\prime}}{\mathrm{d} S}\right|
$$

A contraction between the normal and the transformation operator leads to the Cartesian form of the result.

In effect, the situation is similar to the Euler flow, where one can only impose one condition at the wall- that is, zero normal velocity. It could be added that a single wall boundary condition is also consistent with well-posedness, for it is widely known that only one characteristic enters the flow domain through the wall, which implies that a single condition can be specified there. The outgoing characteristic gives rise to the relation between the normal component of the pressure gradient and the centripetal force term $[35,36]$ and establishes pressure as the only dependent flow variable to be strictly required at the wall. All the remaining variables are extrapolated from the flow solution [35,43].

In principle, the same should hold for the adjoint variables, because they share the same characteristics as the flow, except for the sign reversal. The problem with extrapolating the remaining adjoint variables at the wall is that they have no underlying physics as the flow variables do. As a result, a few different extrapolation schemes have been discussed in the literature, and, among them we have picked one proposed by Reuther [40]. This scheme will be presented in detail as follows for the $2 \mathrm{D}$ test cases.

Functional (b) in Equation (19) is the part of the bilinear concomitant in (14) that corresponds to an integral over the far-field boundary $B_{\infty}$. There, one recognizes in the term $\left(C_{\alpha \beta}^{2} \delta \bar{Q}_{\beta} n^{2}\right)$ the physical part of the flux variation, from Equation (13), in the normal direction to $B_{\infty}$. On accounting for this, that integral may as well be seen as an inner product between the $\Psi_{\alpha}$ and physical variations of the flux that crosses the far-field boundary. On pursuing a similar approach to what is outlined previously, the idea is, first, to constrain the $\delta \bar{Q}_{\beta}$ to the locus of realizable variations, as is determined by the corresponding flow boundary conditions. Then, by assigning zero value to the inner product, one imposes on $\psi_{\alpha}$ the condition that they should be normal to all admissible virtual displacements of the flux vector at the far-field boundary.

$$
\int_{B_{\infty}} \Psi_{\alpha} C_{\alpha \beta}^{2} \delta \bar{Q}_{\beta} n_{2} \mathrm{~d} S=\int_{B_{\infty}} \psi_{\alpha} \beta_{i^{\prime}}^{2} A_{\alpha \beta}^{i^{\prime}} \delta \bar{Q}_{\beta} n_{2} \mathrm{~d} S=\left.0 \Rightarrow \psi_{\alpha}\left(A_{\alpha \beta}^{i^{\prime}} n_{i^{\prime}}\right) \delta \bar{Q}_{\beta}\right|_{B_{\infty}}=0
$$

Here, the definitions of $\psi_{\alpha}$ and Equation (9) have been used to pass from the first to the second form. Use was also made of the fact that, once it has been constrained to be realizable, the $\delta \bar{Q}_{\beta}$ should also be deemed arbitrary-hence, the need for the last equation. In any case, the procedure is better illustrated with the simple example of supersonic flow [38].

At a supersonic inflow section, all flow characteristics enter the domain. It implies that all flow variables are fixed at that boundary and, thus, their variations are identically zero, $\delta \bar{Q}_{\beta}=0$. Because the adjoint characteristics run in the opposite direction, they all leave the domain. As a result, no boundary conditions should be imposed on the $\psi_{\alpha}$ there. For well-posedness, their values should be determined as part of the adjoint solution instead. However, the fact that $\delta \bar{Q}_{\beta}$ is zero ensures that the inner product (24) vanishes at that boundary. Hence, the orthogonality condition holds for any vector $\psi_{\alpha}$.

The exact opposite occurs at a supersonic outflow section. There, all of the flow characteristics leave the domain, whereas the adjoint ones enter it. For the flow, it implies that no boundary conditions should be imposed, and the physical variations $\delta \bar{Q}_{\beta}$ are free. On the other hand, the incoming adjoint characteristics require that all $\psi_{\alpha}$ be prescribed there, for well-posedness. In principle, the only means of ensuring that orthogonality (24) holds is to impose homogeneous boundary conditions on the adjoint variables there, $\psi_{\alpha}=0$. This is the only situation where homogeneous boundary conditions are strictly required. 
Boundary conditions for subsonic flow need more careful consideration, for, in that case, the characteristics run both ways, in and out of the flow domain. Then, the admissible flux variations at the boundaries are only partially constrained. Yet, the procedure remains the same: first, one evaluates the realizable $\delta \bar{Q}_{\beta}$ in each case and then finds out the orthogonal $\psi_{\alpha}$ thereof.

As it has been appropriately discussed by Giles and Pierce [31], the sign reversal of the adjoint characteristics plays a crucial role in ensuring that the equations are well posed. In the context of a Riemann problem, characteristics that enter the domain convey Cauchy data from the boundaries to the solution, whereas those that leave it feed information back from the latter to the former. Hence, to each domain incoming characteristic there must correspond a boundary condition. Conversely, each outgoing characteristic implies that some variation must be allowed for at the boundary.

In a sense, the state variables can be seen as degrees of freedom (DOFs) of the boundary problem. Naturally, their number corresponds to the dimension $N$ of the state space: $N=5$ for 3D flows and $N=4$ in 2D problems. In this context, each boundary condition constrains one DOF at the boundary, whereas each outgoing characteristic corresponds to a DOF that is preserved. The scheme holds for both problems, the Euler flow and its adjoint. However, in view of the sign reversal, to each DOF that is constrained for the flow at a boundary, there corresponds one that is left for the adjoint, and vice versa.

The contribution of this paper is to combine these ideas with a condition of orthogonality, which comes from Equation (24). With regard to the variational problem, the boundary conditions constrain the $\delta \bar{Q}_{\beta}$ to a locus of realizability in state space, where they can effectively be deemed realizable and arbitrary. Within that locus, all virtual displacements are admissible and, by introducing them into Equation (24), one can work out the $\psi_{\alpha}$ that are normal to the locus. The resulting adjoint vector is compatible with all admissible variations at that boundary.

As will be shown, for each DOF that is constrained in a fluid boundary condition, the inner product (24) preserves one DOF in the corresponding adjoint boundary condition. As a result of the symmetry, the balance between constrained and preserved DOFs agrees with the balance between adjoint characteristics that enter or leave the domain. It implies that there will be as many adjoint boundary conditions that satisfy (24), as there will be adjoint characteristics that run into the domain. The number of remaining DOFs will, likewise, be the same as that of outgoing characteristics. Under these conditions, one is able to remove the far-field integral (b) from the sensitivity gradient (19) and also to be consistent with the well-posedness of the adjoint problem.

Before proceeding with the derivation of boundary conditions, however, it is important to note that the adjoint equations (22) are generalized in either form. Therefore, they must hold in any coordinate system and, in particular, in the Cartesian. The same applies to the periodic condition that is imposed on cut planes (16). On the other hand, the wall (23) and far-field (24) boundary conditions have been written for a specific system, where both boundaries are mapped onto constant coordinate planes: $B_{w} \Rightarrow \xi^{2}=0$ and $B_{\infty} \Rightarrow \xi^{2}=1$, respectively. However, they can also be put in Cartesian coordinates, as those equations show.

That opens up the possibility of solving the adjoint problem in the Cartesian system and, thus, to dispense with the coordinate transformation altogether. It all hinges upon one's ability to compute the metric variations $\delta\left(J \beta_{i^{\prime}}^{2}\right)$ that appear in the sensitivity gradient (19) without having to resort to the transformation explicitly. If that is accomplished, then the transformation has fulfilled its prime objective, which was to simplify the derivation, and can be discarded. As it turns out, in 2D applications, one can indeed evaluate those terms at the contour $B_{w}$ without actually computing the transformation. The procedure will be discussed at length in Section 5.3 after the boundary conditions.

\section{2D EULER FLOW, ADJOINT EQUATIONS AND BOUNDARY CONDITIONS}

In view of what was discussed earlier, the adjoint problem will be entirely constructed and solved in Cartesian coordinates. For 2D cases, the Greek subscripts range from 1 to 4 , where 1 refers to continuity, 2 and 3 to linear momentum and 4 to energy, whereas the Latin indices refer to the two dimensions in physical space-for simplicity, we designate them as $x^{1^{\prime}}=x, x^{2^{\prime}}=y$. 
The corresponding flux Jacobian matrices are denoted by $\mathbf{A}^{\mathbf{1}^{\prime}}=\mathbf{A}$ and $\mathbf{A}^{2^{\prime}}=\mathbf{B}$, respectively. Under these circumstances, the adjoint equations become

$$
\frac{\partial \psi_{\alpha}}{\partial t}-A_{\beta \alpha} \frac{\psi_{\beta}}{\partial x}-B_{\beta \alpha} \frac{\psi_{\beta}}{\partial y}=0
$$

For simplicity, the flux Jacobian vector [35] $A_{\beta \alpha}^{i^{\prime}}$ is also assigned a symbolic notation, $A_{\beta \alpha}^{i^{\prime}} \Rightarrow$ $\overline{\mathbf{A}}^{T}=\left(\mathbf{A}^{T}, \mathbf{B}^{T}\right)$.

It is widely known that the flux Jacobian matrices have complete eigensystems and that they can be diagonalized upon their being associated with a given direction $[35,43]$. The normal direction to a boundary is of particular interest, in that regard, for the characteristics associated with it are responsible for all the exchange of information across that boundary. For a general treatment of such boundaries, we pick an arbitrary direction, as defined by the unit vector $\mathbf{k}=\left(k_{x}, k_{y}\right)^{T}$, and the corresponding Jacobian matrix is given by the dot product

$$
-\overline{\mathbf{A}}^{T} \cdot \mathbf{k}=-k_{x} \mathbf{A}^{T}-k_{y} \mathbf{B}^{T}
$$

where the minus sign and the transposition, which appear in Equation (25), have been retained for future convenience. The similarity transformation that diagonalizes the resulting matrix yields

$$
\begin{aligned}
-\boldsymbol{\Lambda} & =-\mathbf{P}^{T}\left(\overline{\mathbf{A}}^{T} \cdot \mathbf{k}\right)\left(\mathbf{P}^{-1}\right)^{T} \\
& =\left(\begin{array}{cccc}
-\left(k_{x} u+k_{y} v\right) & 0 & 0 & 0 \\
0 & -\left(k_{x} u+k_{y} v\right) & 0 & 0 \\
0 & 0 & -\left(k_{x} u+k_{y} v+c\right) & 0 \\
0 & 0 & 0 & -\left(k_{x} u+k_{y} v-c\right)
\end{array}\right)
\end{aligned}
$$

which are obviously the same eigenvalues of the Euler equations but for the sign reversal. Also associated with the direction $\mathbf{k}$, the similarity transformation operators are given by [35]

$$
\begin{gathered}
\mathbf{P}^{T}=\left(\begin{array}{cccc}
1 & u & v & \frac{u^{2}+v^{2}}{2} \\
0 & \rho k_{y} & -\rho k_{x} & \rho\left(u k_{y}-v k_{x}\right) \\
\frac{\rho}{2 c} & \frac{\rho\left(c k_{x}+u\right)}{2 c} & \frac{\rho\left(c k_{y}+v\right)}{2 c} & \frac{\rho}{2}\left(\frac{c}{\gamma-1}+u k_{x}+v k_{y}+\frac{u^{2}+v^{2}}{2 c}\right) \\
\frac{\rho}{2 c} & \frac{\rho\left(v-c k_{x}\right)}{2 c} & \frac{\rho\left(v-c k_{y}\right)}{2 c} & \frac{\rho}{2}\left(\frac{c}{\gamma-1}-u k_{x}-v k_{y}+\frac{u^{2}+v^{2}}{2 c}\right)
\end{array}\right) \\
\left(\mathbf{P}^{-1}\right)^{T}=\left(\begin{array}{cccc}
1-\frac{(\gamma-1)\left(u^{2}+v^{2}\right)}{2 c^{2}} & \frac{v k_{x}-u k_{y}}{\rho} & \frac{(\gamma-1)\left(u^{2}+v^{2}\right)-2 c\left(u k_{x}+v k_{y}\right)}{2 \rho c} & \frac{(\gamma-1)\left(u^{2}+v^{2}\right)+2 c\left(u k_{x}+v k_{y}\right)}{2 \rho c} \\
\frac{(\gamma-1) u}{c^{2}} & \frac{1}{\rho} k_{y} & \frac{c k_{x}-(\gamma-1) u}{\rho c} & \frac{-c k_{x}-(\gamma-1) u}{\rho c} \\
\frac{(\gamma-1) v}{c^{2}} & -\frac{1}{\rho} k_{x} & \frac{c k_{y}-(\gamma-1) v}{\rho c} & \frac{-c k_{y}-(\gamma-1) v}{\rho c} \\
-\frac{(\gamma-1)}{c^{2}} & 0 & \frac{\gamma-1}{\rho c} & \frac{\gamma-1}{\rho c}
\end{array}\right)
\end{gathered}
$$

respectively.

Although the adjoint PDEs cannot be fully diagonalized, one can obtain their characteristic form by left multiplying Equation (25) through by $\mathbf{P}^{T}$. It leads to the expression

$$
\mathbf{P}^{T} \frac{\partial \boldsymbol{\psi}}{\partial t}-\mathbf{P}^{T} \mathbf{A}^{T}\left(\mathbf{P}^{-1}\right)^{T} \mathbf{P}^{T} \frac{\partial \psi}{\partial x}-\mathbf{P}^{T} \mathbf{B}^{T}\left(\mathbf{P}^{-1}\right)^{T} \mathbf{P}^{T} \frac{\partial \boldsymbol{\psi}}{\partial y}=0
$$

Here, it may be added that the product $\left(\mathbf{P}^{T} \delta \boldsymbol{\psi}\right)$ defines adjoint Riemann differentials. Accordingly, on computing the matrix products in Equation (30), one obtains a set of Riemann's equations, or characteristics, of the form 


$$
\sum_{\beta=1}^{N} K_{\alpha \beta}^{t} \frac{\partial \psi_{\beta}}{\partial t}=\sum_{\beta=1}^{N} K_{\alpha \beta}^{x} \frac{\partial \psi_{\beta}}{\partial x}+\sum_{\beta=1}^{N} K_{\alpha \beta}^{y} \frac{\partial \psi_{\beta}}{\partial y}
$$

where $N=4$ in the $2 \mathrm{D}$ cases and the coefficients $K_{\alpha \beta}^{t}, K_{\alpha \beta}^{x}$ and $K_{\alpha \beta}^{y}$ are listed in Table I.

Table I. Coefficients.

\begin{tabular}{|c|c|c|c|}
\hline Coef. & Value & Coef. & Value \\
\hline$K_{11}^{t}$ & 1 & $K_{31}^{x}$ & $\rho\left(u+k_{x} c\right)$ \\
\hline$K_{12}^{t}$ & $u$ & $K_{32}^{x}$ & {$\left[\rho c^{2} k_{y}^{2}+\rho\left(u+k_{x} c\right)^{2}\right]$} \\
\hline$K_{13}^{t}$ & $v$ & $K_{33}^{x}$ & $-\left[\rho c^{2} k_{x} k_{y}-\rho\left(u+k_{x} c\right)\left(v+k_{y} c\right)\right]$ \\
\hline \multirow[t]{2}{*}{$K_{14}^{t}$} & $\frac{\left(u^{2}+v^{2}\right)}{2}$ & $K_{34}^{x}$ & $\begin{array}{l}-\left[\rho c^{2} k_{y}\left(k_{x} v-k_{y} u\right)+\right. \\
-\frac{\rho}{2}\left(u+k_{x} c\right)\left(u^{2}+v^{2}\right)+\end{array}$ \\
\hline & & & $\left.-\rho c\left(u+k_{x} c\right)\left(k_{x} u+k_{y} v\right)-\frac{\rho c^{2}\left(u+k_{x} c\right)}{\gamma-1}\right]$ \\
\hline$K_{21}^{t}$ & 0 & $K_{41}^{x}$ & $\rho\left(u-k_{x} c\right)$ \\
\hline$K_{22}^{t}$ & $\rho k_{y}$ & $K_{42}^{x}$ & {$\left[\rho c^{2} k_{y}^{2}+\rho\left(u-k_{x} c\right)^{2}\right]$} \\
\hline$K_{23}^{t}$ & $-\rho k_{x}$ & $K_{43}^{x}$ & $-\left[\rho c^{2} k_{x} k_{y}-\rho\left(u-k_{x} c\right)\left(v-k_{y} c\right)\right]$ \\
\hline \multirow[t]{2}{*}{$K_{24}^{t}$} & $\rho\left(k_{y} u-k_{x} v\right)$ & $K_{44}^{x}$ & {$\left[\rho c^{2} k_{y}\left(k_{x} v-k_{y} u\right)+\right.$} \\
\hline & & & $\begin{array}{c}+\frac{\rho}{2}\left(u-k_{x} c\right)\left(u^{2}+v^{2}\right)+ \\
\left.-\rho c\left(u-k_{x} c\right)\left(k_{x} u+k_{y} v\right)+\frac{\rho c^{2}\left(u-k_{x} c\right)}{\gamma-1}\right]\end{array}$ \\
\hline$K_{31}^{t}$ & $\rho$ & $K_{11}^{y}$ & $v$ \\
\hline$K_{32}^{t}$ & $\rho\left(u+k_{x} c\right)$ & $K_{12}^{y}$ & $u v$ \\
\hline$K_{33}^{t}$ & $\rho\left(v+k_{y} c\right)$ & $K_{13}^{y}$ & $v^{2}$ \\
\hline$K_{34}^{t}$ & $\begin{array}{c}{\left[\frac{\rho c^{2}}{\gamma-1}+\rho c\left(k_{x} u+k_{y} v\right)+\right.} \\
\left.+\frac{\rho}{2}\left(u^{2}+v^{2}\right)\right]\end{array}$ & $K_{14}^{y}$ & $\frac{v\left(u^{2}+v^{2}\right)}{2}$ \\
\hline$K_{41}^{t}$ & $\rho$ & $K_{21}^{y}$ & $-\rho k_{x}$ \\
\hline$K_{42}^{t}$ & $\rho\left(u-k_{x} c\right)$ & $K_{22}^{y}$ & $-\rho\left(k_{x} u-k_{y} v\right)$ \\
\hline$K_{43}^{t}$ & $\rho\left(v-k_{y} c\right)$ & $K_{23}^{y}$ & $-2 \rho k_{x} v$ \\
\hline \multirow[t]{2}{*}{$K_{44}^{t}$} & {$\left[\frac{\rho c^{2}}{\gamma-1}-\rho c\left(k_{x} u+k_{y} v\right)+\right.$} & $K_{24}^{y}$ & {$\left[\rho k_{y} u v-\frac{\rho k_{x}\left(u^{2}+3 v^{2}\right)}{2}-\frac{\rho c^{2} k_{x}}{\gamma-1}\right]$} \\
\hline & $\left.+\frac{\rho}{2}\left(u^{2}+v^{2}\right)\right]$ & & \\
\hline$K_{11}^{x}$ & $u$ & $K_{31}^{y}$ & $\rho\left(v+k_{y} c\right)$ \\
\hline$K_{12}^{x}$ & $u^{2}$ & $K_{32}^{y}$ & $-\left[\rho c^{2} k_{x} k_{y}-\rho\left(u+k_{x} c\right)\left(v+k_{y} c\right)\right]$ \\
\hline$K_{13}^{x}$ & $u v$ & $K_{33}^{y}$ & {$\left[\rho c^{2} k_{x}^{2}+\rho\left(v+k_{y} c\right)^{2}\right]$} \\
\hline \multirow[t]{3}{*}{$K_{14}^{x}$} & $\frac{u\left(u^{2}+v^{2}\right)}{2}$ & $K_{34}^{y}$ & $-\left[\rho c^{2} k_{x}\left(k_{y} u-k_{x} v\right)-\frac{\rho c^{2}\left(v+k_{y} c\right)}{\gamma-1}+\right.$ \\
\hline & & & $-\rho c\left(v+k_{y} c\right)\left(k_{x} u+k_{y} v\right)+$ \\
\hline & & & $-\frac{\rho}{2}\left(v+k_{y} c\right)\left(u^{2}+v^{2}\right)$ \\
\hline$K_{21}^{x}$ & $\rho k_{y}$ & $K_{41}^{y}$ & $\rho\left(v-k_{y} c\right)$ \\
\hline$K_{22}^{x}$ & $2 \rho k_{y} u$ & $K_{42}^{y}$ & $-\left[\rho c^{2} k_{x} k_{y}+\rho\left(u-k_{x} c\right)\left(v-k_{y} c\right)\right]$ \\
\hline$K_{23}^{x}$ & $-\rho\left(k_{x} u-k_{y} v\right)$ & $K_{43}^{y}$ & {$\left[\rho c^{2} k_{x}^{2}-\rho\left(v-k_{y} c\right)^{2}\right]$} \\
\hline \multirow[t]{3}{*}{$K_{24}^{x}$} & $-\left[\rho k_{x} u v-\frac{\rho k_{y}\left(3 u^{2}+v^{2}\right)}{2}-\frac{\rho c^{2} k_{y}}{\gamma-1}\right.$ & $K_{44}^{y}$ & $-\left[\rho c^{2} k_{x}\left(k_{y} u-k_{x} v\right)-\frac{\rho c^{2}\left(v-k_{y} c\right)}{\gamma-1}+\right.$ \\
\hline & & & $+\rho c\left(v-k_{y} c\right)\left(k_{x} u+k_{y} v\right)+$ \\
\hline & & & $-\frac{\rho}{2}\left(v-k_{y} c\right)\left(u^{2}+v^{2}\right)$ \\
\hline
\end{tabular}


Just as the original equation (25), the set (31) is linear. Only, the coefficients are given by less familiar algebraic expressions. Different from the former, however, each one of the preceding equations $(\alpha)$ is intrinsically associated with a specific characteristic velocity, as given by the matrix $\Lambda$ in Equation (27). Ultimately, on choosing the vector $\mathbf{k}$ normal to the domain boundaries, those velocities determine which of the adjoint characteristics enter or leave the flow domain. Therefore, they are essential to set up the conditions at each boundary.

As was discussed in Section 3.1, the domain incoming characteristics are replaced by boundary conditions, whereas the outgoing ones feed domain information back to the boundary, thus completing the Riemann problem. The equations that represent the latter are picked out from (31) for each case.

Next, the topic is considered at length for each of the boundaries.

\subsection{Solid wall}

The $2 \mathrm{D}$ contour of the body can be specified as a level curve, such as $\eta(x, y)=0$, in a plane mapping of type $(x, y) \rightleftharpoons(\xi, \eta)$. Alternatively, it can be cast in parametric form as

$$
\eta(x, y)=0 \Leftrightarrow\left\{\begin{array}{l}
x=x(\xi, 0) \\
y=y(\xi, 0)
\end{array}\right.
$$

In the first case, the normal unit vector can be obtained by simply making $\mathbf{n}=\nabla \eta /\|\nabla \eta\|$. The corresponding expression for the second case may be derived from the first by means of the relations $\eta_{, x}=-y_{, \xi} / J$ and $\eta_{, y}=x_{, \xi} / J$, which are obtained on inverting the operator $\beta_{j^{\prime}}^{i}$ to compute $\beta_{k}^{i^{\prime}}$. As a result, the normal vector becomes

$$
n_{j^{\prime}} \Rightarrow\left(\begin{array}{c}
k_{x} \\
k_{y}
\end{array}\right)=\frac{1}{\sqrt{\eta_{, x}^{2}+\eta_{, y}^{2}}}\left(\begin{array}{c}
\eta_{, x} \\
\eta_{, y}
\end{array}\right)=\frac{1}{\sqrt{x_{, \xi}^{2}+y_{, \xi}^{2}}}\left(\begin{array}{c}
-y_{, \xi} \\
x_{, \xi}
\end{array}\right)
$$

in the Cartesian system. The term that multiplies the vector on the RHS can be recognized to be an element of the metric tensor $g_{i j}$ in transformed space: $g_{11}=x_{, \xi}^{2}+y_{, \xi}^{2}$. In any case, the result yields a fairly simple expression for the vector covariant components in the transformed plane.

$$
n_{i}=n_{j^{\prime}} \beta_{i}^{j^{\prime}}=\frac{1}{\sqrt{g_{11}}}\left(\begin{array}{l}
0 \\
J
\end{array}\right)
$$

Before proceeding with the derivation, it should be noted that both $\mathbf{n}$ and the metric term $g_{11}$ can be evaluated at the wall solely on the basis of the parametric representation of the contour.

The wall boundary condition is given by Equation (23). It depends on the specification of a metric term, namely the area element ratio $\left|\mathrm{d} S^{\prime} / \mathrm{d} S\right|$. In parametric form (32), one has $\mathrm{d} S=\mathrm{d} \xi$. Besides, $\mathrm{d} S^{\prime}$ and $\mathrm{d} S$ are clearly parallel: $\mathrm{d} S^{\prime} \| \mathrm{d} S$. However, the ratio between the two of them comes from the arc length element $[44,45]$

$$
\left|\frac{\mathrm{d} S^{\prime}}{\mathrm{d} S}\right| \mathrm{d} S=\left|\frac{\mathrm{d} S^{\prime}}{\mathrm{d} \xi}\right| \mathrm{d} \xi=\sqrt{1+\left(\frac{\mathrm{d} y}{\mathrm{~d} x}\right)_{B_{w}}^{2}} x_{, \xi} \mathrm{d} \xi=\sqrt{x_{, \xi}^{2}+y_{, \xi}^{2}} \mathrm{~d} \xi=\sqrt{g_{11}} \mathrm{~d} \xi
$$

Hence, the solid wall boundary condition simply becomes

$$
k_{x} \psi_{2}+k_{y} \psi_{3}=\frac{\partial g}{\partial p} \sqrt{g_{11}}
$$

As was discussed previously, the adjoint solution is computed on the basis of a stationary flow field. Therefore, by differentiating Equation (36) with respect to time, one obtains

$$
k_{x} \frac{\partial \psi_{2}}{\partial t}+k_{y} \frac{\partial \psi_{3}}{\partial t}=0
$$

${ }^{\sharp}$ A shorthand notation is used here to denote partial derivatives of metric terms ()$_{, \xi}=\partial() / \partial \xi$ and ()$_{, x}=\partial() / \partial x$. 
This expression is used next to simplify the equation of the characteristic that leaves the domain through the solid wall boundary.

The normal velocity vanishes identically at the wall $\left(k_{x} u+k_{y} v=0\right)$, which implies that the first two characteristic velocities in Equation (27) are zero. They cannot transfer any Cauchy data across that boundary. On the other hand, the third and fourth ones become $-c$ and $+c$, respectively. Therefore, the third characteristic leaves the domain, while the fourth one enters it. In view of these results, the third characteristic equation in (31) should be solved for the adjoint variables at the wall, whereas the fourth one should be replaced by condition (36). Two DOFs still remain because of the first and second characteristics. In principle, then, these should be extrapolated from the domain.

On taking the third characteristic from (31) and factoring the zero normal velocity in it, as well as Equation (37), one obtains a simplified form of the equation that should be solved for the $\psi_{k}$ at the wall. The equation reads

$$
\begin{aligned}
\frac{\partial \psi_{1}}{\partial t}+u \frac{\partial \psi_{2}}{\partial t}+v \frac{\partial \psi_{3}}{\partial t}+h_{o} \frac{\partial \psi_{4}}{\partial t}= & \left(v+k_{y} c\right) \frac{\partial \psi_{1}}{\partial y}+\left[c^{2} k_{y}^{2}+\left(c k_{x}+u\right)^{2}\right] \frac{\partial \psi_{2}}{\partial x}+ \\
& -\left[c^{2} k_{x} k_{y}-\left(c k_{x}+u\right)\left(c k_{y}+v\right)\right]\left(\frac{\partial \psi_{2}}{\partial y}+\frac{\partial \psi_{3}}{\partial x}\right)+ \\
& +\left(u+k_{x} c\right) \frac{\partial \psi_{1}}{\partial x}+\left[c^{2} k_{x}^{2}+\left(c k_{y}+v\right)^{2}\right] \frac{\partial \psi_{3}}{\partial y}+ \\
& -\left[c^{2} k_{y}\left(k_{x} v-k_{y} u\right)-\left(u+k_{x} c\right) h_{o}\right] \frac{\partial \psi_{4}}{\partial x}+ \\
& -\left[c^{2} k_{x}\left(k_{y} u-k_{x} v\right)-\left(v+k_{y} c\right) h_{o}\right] \frac{\partial \psi_{4}}{\partial y}
\end{aligned}
$$

where the symbol $h_{o}$ stands for the specific stagnation enthalpy.

Equations (38) and (36) represent all the Cauchy data that cross the solid wall. Together, they should form the set of Riemann equations at that boundary. The problem with that set is the presence of all four adjoint variables in Equation (38). Had the simplified characteristic equation been rid of $\psi_{1}$ and $\psi_{4}$, then one would have a closed set to solve for $\psi_{2}$ and $\psi_{3}$. In that case, $\psi_{1}$ and $\psi_{4}$ would not be part of the Cauchy problem at the wall, and thus, they would be determined by numerical extrapolation alone. The situation would then be similar to the corresponding flow boundary problem.

As it stands, however, the set cannot be solved for the $\psi_{k}$ at the wall. Instead, one must rely on Equation (36) and an extrapolation scheme. In that regard, the aforementioned Reuther's [40] scheme suits our applications very well. It adopts zero-order extrapolations for $\psi_{1}$ and $\psi_{4}$ and divides (36) into two conditions between $\psi_{2}$ and $\psi_{3}$ so that their sum recovers the original equation. On representing ghost cells values by $\psi_{k}^{-}$and the corresponding values in domain neighboring cells by $\psi_{k}^{+}$, the equations read

$$
\left\{\begin{array}{l}
\psi_{1}^{-}=\psi_{1}^{+} \\
\psi_{2}^{-}=\psi_{2}^{+}+\frac{2 k_{x}}{k_{x}^{2}+k_{y}^{2}}\left(\frac{\partial g}{\partial p} \sqrt{g_{11}}-k_{x} \Psi_{2}^{+}-k_{y} \Psi_{3}^{+}\right) \\
\psi_{3}^{-}=\psi_{3}^{+}+\frac{2 k_{y}}{k_{x}^{2}+k_{y}^{2}}\left(\frac{\partial g}{\partial p} \sqrt{g_{11}}-k_{x} \Psi_{2}^{+}-k_{y} \Psi_{3}^{+}\right) \\
\psi_{4}^{-}=\psi_{4}^{+}
\end{array}\right.
$$

where the condition (36) is met at the wall boundary in terms of the average

$$
k_{x} \frac{\left(\psi_{2}^{-}+\psi_{2}^{+}\right)}{2}+k_{y} \frac{\left(\psi_{3}^{-}+\psi_{3}^{+}\right)}{2}=\frac{\partial g}{\partial p} \sqrt{g_{11}}
$$

This scheme has been amply verified in the literature and by ourselves [36,46]. It has shown to lead to estimates of the sensitivity gradient that are close to those obtained by finite differences, within reasonable accuracy levels [40]. Therefore, it is fully consistent with what is expected of the adjoint method. 


\subsection{Inflow}

At a subsonic inflow boundary, there is only one adjoint characteristic that enters the domain. By taking $\mathbf{n}$ to point inward, it will be the one that is associated with the eigenvalue $-(\mathbf{u} \cdot \mathbf{n}-c)$ in Equation (27), that is, the fourth PDE in (31). Hence, that equation must be replaced by a boundary condition, which should come from Equation (24), as a requirement of realizability

$$
\left.\psi_{\alpha}\left(A_{\alpha \beta}^{i^{\prime}} n_{i^{\prime}}\right) \delta \bar{Q}_{\beta}\right|_{B_{\infty}}=0
$$

Together with the first three PDEs from set (31), this condition forms the Riemann problem at that boundary. In order to fully specify it, one must first find out which virtual displacements $\delta \bar{Q}_{\beta}$ are admissible there.

At a subsonic entrance, the flow boundary conditions require that the velocity direction $\theta$, along with the stagnation pressure $p_{o}$ and temperature $T_{o}$, be prescribed. It clearly implies that the corresponding variations are null: $\delta \theta=\delta p_{o}=\delta T_{o}=0$. In terms of state variables $Q_{\beta}$, these quantities are given by

$$
\left\{\begin{aligned}
\tan (\theta) & =\frac{Q_{3}}{Q_{2}} \\
T_{o} & =\frac{(\gamma-1)}{2 \gamma R Q_{1}^{2}}\left[2 \gamma Q_{1} Q_{4}-(\gamma-1)\left(Q_{2}^{2}+Q_{3}^{2}\right)\right] \\
p_{o} & =\frac{(\gamma-1)\left[2 Q_{1} Q_{4}-\left(Q_{2}^{2}+Q_{3}^{2}\right)\right]}{2 Q_{1}}\left\{1+\frac{\left(Q_{2}^{2}+Q_{3}^{2}\right)}{\gamma\left[2 Q_{1} Q_{4}-\left(Q_{2}^{2}+Q_{3}^{2}\right)\right]}\right\}^{\frac{\gamma}{(\gamma-1)}}
\end{aligned}\right.
$$

By computing $\delta \theta, \delta p_{o}$ and $\delta T_{o}$ in terms of the variations $\delta Q_{\beta}$, and by equating them to zero, one obtains a linear set of three equations. Here, it must be recalled that $\delta Q_{\beta}$ and $\delta \bar{Q}_{\beta}$ are equivalent with regard to realizability. Then on replacing the former by the latter, one can solve the set for three of them in terms of the fourth one. Whichever variation is chosen as the independent one, the resulting expressions will represent the locus of realizability in state space. For instance, on picking $\delta \bar{Q}_{1}$ to be independent, the procedure yields

$$
\delta \bar{Q}_{\beta} \Rightarrow\left(\begin{array}{c}
1 \\
\frac{u}{2}\left[2-\gamma+\gamma^{2}-\frac{2 e_{t}(\gamma-1) \gamma}{\left(u^{2}+v^{2}\right)}\right] \\
\frac{v}{2}\left[2-\gamma+\gamma^{2}-\frac{2 e_{t}(\gamma-1) \gamma}{\left(u^{2}+v^{2}\right)}\right] \\
\frac{1}{2}\left[-2 e_{t}(\gamma-2) \gamma+(\gamma-1)^{2}\left(u^{2}+v^{2}\right)\right]
\end{array}\right) \delta \bar{Q}_{1}
$$

Then, on substituting vector (43) for the corresponding term in Equation (41), one obtains the equation

$$
\begin{aligned}
\delta \bar{Q}_{1}\left\{\psi_{1}[\right. & \left.2-\gamma+\gamma^{2}-\frac{2 \gamma e_{t}(\gamma-1)}{u^{2}+v^{2}}\right] \frac{\left(k_{x} u+k_{y} v\right)}{2}+\psi_{2}\left[\frac{\left(2-\gamma+\gamma^{2}\right) k_{x} u^{2}}{2}+\right. \\
& \left.+\frac{2\left(1-\gamma+\gamma^{2}\right) k_{y} u v-(\gamma-1) \gamma k_{x} v^{2}}{2}-\frac{2 \gamma e_{t}(\gamma-1)\left[2 k_{y} u v+k_{x}\left(u^{2}-v^{2}\right)\right]}{2\left(u^{2}+v^{2}\right)}\right]+ \\
& +\psi_{3}\left[\frac{-\gamma(\gamma-1) k_{y} u^{4}+2 k_{y} u^{2} v^{2}+2\left(1-\gamma+\gamma^{2}\right) k_{x}\left(u^{3} v+u v^{3}\right)+}{2\left(u^{2}+v^{2}\right)}+\right. \\
& \left.+\frac{\left(2-\gamma+\gamma^{2}\right) k_{y} v^{4}+2 \gamma e_{t}(\gamma-1)\left[k_{y}\left(u^{2}-v^{2}\right)-2 k_{x} u v\right]}{2\left(u^{2}+v^{2}\right)}\right]+ \\
& -\psi_{4}\left[\frac{\left(k_{x} u+k_{y} v\right)\left[(\gamma-1)\left(u^{2}+v^{2}\right)\right]\left[\left(2-\gamma+\gamma^{2}\right)\left(u^{2}+v^{2}\right)-2 \gamma e_{t}(\gamma-1)\right]}{4\left(u^{2}+v^{2}\right)}+\right. \\
& \left.\left.-\frac{\left(k_{x} u+k_{y} v\right) 2 \gamma e_{t}\left[\left(2-\gamma+\gamma^{2}\right)\left(u^{2}+v^{2}\right)-2 \gamma e_{t}(\gamma-1)\right]}{4\left(u^{2}+v^{2}\right)}\right]\right\}=0
\end{aligned}
$$

where the $\psi_{\alpha}$ have been collected for clarity. 
In the preceding equation, $\delta \bar{Q}_{1}$ is realizable, but it is otherwise arbitrary. Therefore, the only means for one to drive Equation (44) to zero is to require its coefficient, that is, the term within braces, to vanish identically. That leads to a single linear equation, which involves all four $\psi_{\alpha}$. It can be written in a simplified form as

$$
C_{1} \psi_{1}+C_{2} \psi_{2}+C_{3} \psi_{3}+C_{4} \psi_{4}=0
$$

where the coefficients are given by

$$
\left\{\begin{array}{l}
C_{1}=\left[2-\gamma+\gamma^{2}-\frac{2 \gamma e_{t}(\gamma-1)}{u^{2}+v^{2}}\right] \frac{\left(k_{x} u+k_{y} v\right)}{2} \\
C_{2}=\frac{\left(2-\gamma+\gamma^{2}\right) k_{x} u^{2}+2\left(1-\gamma+\gamma^{2}\right) k_{y} u v}{2}-\frac{(\gamma-1) \gamma k_{x} v^{2}}{2}-\frac{2 \gamma e_{t}(\gamma-1)\left[2 k_{y} u v+k_{x}\left(u^{2}-v^{2}\right)\right]}{2\left(u^{2}+v^{2}\right)} \\
C_{3}=\frac{-\gamma(\gamma-1) k_{y} u^{4}+2 k_{y} u^{2} v^{2}}{2\left(u^{2}+v^{2}\right)}+\frac{2\left(1-\gamma+\gamma^{2}\right) k_{x}\left(u^{3} v+u v^{3}\right)+\left(2-\gamma+\gamma^{2}\right) k_{y} v^{4}+2 \gamma e_{t}(\gamma-1)\left[k_{y}\left(u^{2}-v^{2}\right)-2 k_{x} u v\right]}{2\left(u^{2}+v^{2}\right)} \\
C_{4}=-\frac{\left(k_{x} u+k_{y} v\right)\left[(\gamma-1)\left(u^{2}+v^{2}\right)-2 \gamma e_{t}\right]\left[\left(2-\gamma+\gamma^{2}\right)\left(u^{2}+v^{2}\right)-2 \gamma e_{t}(\gamma-1)\right]}{4\left(u^{2}+v^{2}\right)}
\end{array}\right.
$$

Equation (45) is the boundary condition to be imposed on the adjoint variables, and it may also be seen as a compatibility relation they should meet at the inflow boundary. Here again, because the adjoint problem is based on a stationary flow solution, the coefficients in (46) are independent of time. Therefore, Equation (45) remains valid when the $\psi_{\alpha}$ are differentiated with respect to time. Then, on joining it with the first three characteristic equations from (31), one obtains the complete linear set for the corresponding Riemann problem.

$$
\left\{\begin{array}{c}
K_{11}^{t} \frac{\partial \psi_{1}}{\partial t}+K_{12}^{t} \frac{\partial \psi_{2}}{\partial t}+K_{13}^{t} \frac{\partial \psi_{3}}{\partial t}+K_{14}^{t} \frac{\partial \psi_{4}}{\partial t}=R_{1} \\
K_{21}^{t} \frac{\partial \psi_{1}}{\partial t}+K_{22}^{t} \frac{\partial \psi_{2}}{\partial t}+K_{23}^{t} \frac{\partial \psi_{3}}{\partial t}+K_{24}^{t} \frac{\partial \psi_{4}}{\partial t}=R_{2} \\
K_{31}^{t} \frac{\partial \psi_{1}}{\partial t}+K_{32}^{t} \frac{\partial \psi_{2}}{\partial t}+K_{33}^{t} \frac{\partial \psi_{3}}{\partial t}+K_{34}^{t} \frac{\partial \psi_{4}}{\partial t}=R_{3} \\
C_{1} \frac{\partial \psi_{1}}{\partial t}+C_{2} \frac{\partial \psi_{2}}{\partial t}+C_{3} \frac{\partial \psi_{3}}{\partial t}+C_{4} \frac{\partial \psi_{4}}{\partial t}=0
\end{array}\right.
$$

where the spacial derivatives from the RHS of (31) have all been collected up in the $R_{\alpha}$ terms. The resulting set is amenable to numerical integration by explicit time stepping.

With regard to the discussion on boundary conditions, back in Section 3.1, it is worth noting that, for each one of the three DOFs that are constrained in the flow problem, a corresponding DOF is preserved in the adjoint problem. That symmetry agrees exactly with the balance between the domain incoming and outgoing characteristics for both problems.

\subsection{Outflow}

In the case of an outflow boundary, the adjoint problem has three domain incoming characteristics. They are the ones that correspond to the first two and the fourth eigenvalues in (27), and their equations in (31) must be replaced by boundary conditions. Hence, it is only the characteristic associated with $-(\mathbf{u} \cdot \mathbf{n}+c)$ that needs actually be solved.

Three boundary conditions are required from Equation (24), or from (41) for that matter. Just as in the previous case, these conditions should come as a result of realizability constraints that are imposed on $\delta \bar{Q}_{\alpha}$. In the applications of interest here, the static pressure is the only quantity that is fixed at an outflow boundary. On casting it in terms of the state variables, it yields

$$
p=(\gamma-1)\left(Q_{4}-\frac{\left(Q_{2}^{2}+Q_{3}^{2}\right)}{2 Q_{1}}\right)
$$


By computing its first variation and setting it at zero $(\delta p=0)$, and on recalling that, as far as realizability is concerned, $\delta \bar{Q}_{\alpha} \Leftrightarrow \delta Q_{\alpha}$, one obtains

$$
\delta \bar{Q}_{4}=u \delta \bar{Q}_{2}+v \delta \bar{Q}_{3}-\frac{\left(u^{2}+v^{2}\right)}{2} \delta \bar{Q}_{1}
$$

which preserves three DOFs: $\delta \bar{Q}_{1}, \delta \bar{Q}_{2}$ and $\delta \bar{Q}_{3}$.

Equation (49) represents the locus of realizable variations for the outflow boundary. Again, on substituting it for the corresponding variations in Equation (41), it leads to

$$
\begin{aligned}
& \delta \bar{Q}_{1}\left\{\left(k_{x} u+k_{y} v\right)\left(-u \psi_{2}-v \psi_{3}\right)+\frac{\left(k_{x} u+k_{y} v\right)}{2} \psi_{4}\left[(\gamma-2)\left(u^{2}+v^{2}\right)-2 \gamma e_{t}\right]\right\}+ \\
& \quad+\delta \bar{Q}_{2}\left\{k_{x}\left(\psi_{1}+v \psi_{3}\right)+\psi_{2}\left(u+k_{x} u+k_{y} v\right)+\right. \\
& \left.\quad+\psi_{4}\left[k_{x} \gamma e_{t}+u\left(k_{x} u+k_{y} v\right)+\frac{(\gamma-1) k_{x}\left(u^{2}+v^{2}\right)}{2}\right]\right\}+ \\
& \quad+\delta \bar{Q}_{3}\left\{k_{y}\left(\psi_{1}+u \psi_{2}\right)+\psi_{3}\left(v+k_{x} u+k_{y} v\right)+\right. \\
& \left.\quad+\psi_{4}\left[k_{y} \gamma e_{t}+v\left(k_{x} u+k_{y} v\right)-\frac{(\gamma-1) k_{y}\left(u^{2}+v^{2}\right)}{2}\right]\right\}=0
\end{aligned}
$$

Naturally, the $\delta \bar{Q}_{1}, \delta \bar{Q}_{2}$ and $\delta \bar{Q}_{3}$ are arbitrary, albeit realizable. Therefore, the only means of driving Equation (50) to zero is to require their coefficients to vanish identically. That leads to three boundary conditions, involving all four $\psi_{\alpha}$. They can be written in a simplified form as

$$
\left\{\begin{array}{l}
\psi_{1}=\frac{1}{2}\left[2 e_{t} \gamma-(\gamma-1)\left(u^{2}+v^{2}\right)\right] \psi_{4}=C_{1} \psi_{4} \\
\psi_{2}=\left[\frac{-2 e_{t} \gamma k_{x}+(\gamma-2) k_{x} u^{2}-2 k_{y} u v+\gamma k_{x} v^{2}}{2\left(k_{x} u+k_{y} v\right)}\right] \psi_{4}=C_{2} \psi_{4} \\
\psi_{3}=\left[\frac{-2 e_{t} \gamma k_{y}+(\gamma-2) k_{y} v^{2}-2 k_{x} u v+\gamma k_{y} u^{2}}{2\left(k_{x} u+k_{y} v\right)}\right] \psi_{4}=C_{3} \psi_{4}
\end{array}\right.
$$

Just as in the previous case, all of the previous coefficients are based on a steady flow solution, and thus, they are independent of time. As a result, these equations still hold when the $\psi_{\alpha}$ are differentiated with respect to time.

The complete set for the Riemann problem at the outflow boundary is then obtained by assembling (51) with the equation of the third characteristic from (31)

$$
\begin{cases}\frac{\partial \psi_{1}}{\partial t}-C_{1} \frac{\partial \psi_{4}}{\partial t} & =0 \\ \frac{\partial \psi_{2}}{\partial t}-C_{2} \frac{\partial \psi_{4}}{\partial t} & =0 \\ \frac{\partial \psi_{3}}{\partial t}-C_{3} \frac{\partial \psi_{4}}{\partial t} & =0 \\ \left(\sum_{\beta=1}^{3} K_{3 \beta}^{t} C_{\beta}+K_{34}^{t}\right) \frac{\partial \psi_{4}}{\partial t} & =R_{3}\end{cases}
$$

Similar to the previous case, the spacial derivatives in the RHS of the third equation from (31) have been collected up in the $R_{3}$ term. The resulting set is also amenable to numerical integration by explicit time stepping.

With respect to the relation between the flow and adjoint boundary conditions, from Section 3.1, the situation here is similar to the previous case too, for a single DOF is constrained in the flow problem and a single DOF is preserved in the adjoint. Here again, the symmetry agrees exactly with the balance between the domain incoming and outgoing characteristics for both problems. 


\section{ON COMPARING THE TWO APPROACHES}

A comparison between the previous treatment of the boundary problem and that by Giles and Pierce [31] would be instructive. In the cases of solid walls and of permeable boundaries in supersonic flow, both approaches lead to the same expressions. However, the differences are rather significant for permeable boundaries in subsonic flow. For them, a comparison is drawn on the basis of the linearized form of the boundary conditions, Equations (43), (45), (49) and (51). To derive the corresponding expressions from those authors' work, we present a brief account of the relevant material - the reader is referred to the original paper [31] for further details.

In their approach, the linearized flow equations and the objective functional, which constitute the so-called primal problem, are cast in the form

$$
\left\{\begin{array}{c}
\delta I=(g, \delta \overline{\mathbf{Q}})_{D}+(h, \mathbf{C} \delta \overline{\mathbf{Q}})_{\partial D} \\
\mathbf{L} \delta \overline{\mathbf{Q}}=f \quad \text { in } \quad D \\
\mathbf{B} \delta \overline{\mathbf{Q}}=e \quad \text { on } \quad \partial D
\end{array}\right.
$$

where the terms in parentheses in the first equation represent inner products-the first one is an integral over the flow domain and the second an integral over its boundary. The symbols $\mathbf{L}$ and B stand for linearized operators of the flow equations and boundary conditions, respectively. The adjoint or dual problem, in turn, is then given by

$$
\left\{\begin{array}{l}
\delta I=(\Psi, f)_{D}+\left(\mathbf{C}^{*} \Psi, e\right)_{\partial D} \\
\mathbf{L}^{*} \Psi=g \quad \text { in } \quad D \\
\mathbf{B}^{*} \Psi=h \quad \text { on } \partial D
\end{array}\right.
$$

here, the symbols $\mathbf{L}^{*}$ and $\mathbf{B}^{*}$ represent the adjoint equations and their boundary conditions, respectively.

The equivalence between the two forms of the variation $\delta I$ hinges upon a decomposition of the bilinear concomitant from Equation (14). To be more specific, it only concerns the physical part of the flux variation in Equation (13), which is then written in terms of the flux Jacobian matrix associated with the normal direction to $\partial D$, as it appears in Equation (24). The proposed decomposition is as follows:

$$
\int_{\partial D} \Psi^{T}\left[\mathbf{A}-\left(\mathbf{B}^{*}\right)^{T} \mathbf{C}+\left(\mathbf{C}^{*}\right)^{T} \mathbf{B}\right] \delta \overline{\mathbf{Q}} \mathrm{d} S=0
$$

Because $\delta \overline{\mathbf{Q}}$ is arbitrary, the nullity of the integral can only be ensured by driving the expression within square brackets to zero. That, in turn, can be achieved by defining the matrix operators $\mathbf{T}$ and $\mathbf{T}^{*}$ and by computing their product in the form

$$
\mathbf{T} \equiv\left(\frac{\mathbf{B}}{\mathbf{C}}\right) \quad ; \quad \mathbf{T}^{*} \equiv\left(\frac{-\mathbf{C}^{*}}{\mathbf{B}^{*}}\right) \quad \Rightarrow \quad \mathbf{A}=\left(\mathbf{T}^{*}\right)^{T} \mathbf{T}
$$

These matrix operators can also be cast in terms of characteristic (Riemann) variables by means of the same similarity transformation that diagonalizes $\mathbf{A}$

$$
\left.\begin{array}{l}
\mathbf{T}_{c}=\mathbf{T P} \\
\mathbf{T}_{c}^{* T}=\mathbf{P}^{-1} \mathbf{T}^{* T}
\end{array}\right\} \Rightarrow \Lambda=\left(\mathbf{T}_{c}^{*}\right)^{T} \mathbf{T}_{c} \quad \Rightarrow \mathbf{T}_{c}=\left(\frac{\mathbf{B}_{c}}{\mathbf{C}_{c}}\right) \quad ; \quad \mathbf{T}_{c}^{*}=\left(\frac{-\mathbf{C}_{c}^{*}}{\mathbf{B}_{c}^{*}}\right)
$$

The preceding procedure puts the boundary operators themselves in terms of characteristic variables through $\mathbf{B}_{c}$ and $\mathbf{B}_{c}^{*}$, which is precisely the way they are presented in [31]. Hence, one can make use of the inverse similarity transformation to recover the corresponding conservative forms. That is carried out as follows for the purpose of comparing linearized boundary conditions. 


\subsection{Inflow}

At a subsonic inflow boundary, the boundary operators of the primal problem are given by [31] in terms of characteristic variables:

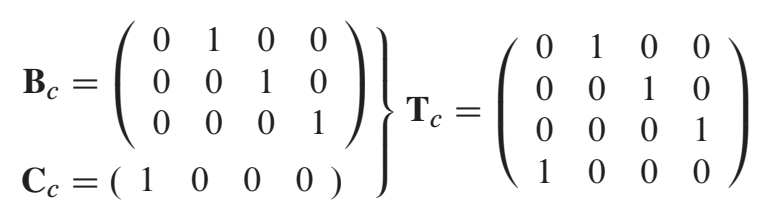

The inverse similarity transformation leads to

$$
\mathbf{T}=\left(\begin{array}{cccc}
\frac{k_{x} v-k_{y} u}{\rho} & \frac{k_{y}}{\rho} & -\frac{k_{x}}{\rho} & 0 \\
\frac{(\gamma-1) \mathbf{u} \cdot \mathbf{u}-2 c \mathbf{k} \cdot \mathbf{u}}{2 c \rho} & \frac{c k_{x}-(\gamma-1) u}{c \rho} & \frac{c k_{y}-(\gamma-1) v}{c \rho} & \frac{(\gamma-1)}{c \rho} \\
\frac{(\gamma-1) \mathbf{u} \cdot \mathbf{u}+2 c \mathbf{k} \cdot \mathbf{u}}{2 c \rho} & -\frac{(\gamma-1) u+c k_{x}}{c \rho} & -\frac{(\gamma-1) v+k_{y}}{c \rho} & \frac{(\gamma-1)}{c \rho} \\
\frac{2 c^{2}-(\gamma-1) \mathbf{u} \cdot \mathbf{u}}{2 c^{2}} & \frac{(\gamma-1) u}{c^{2}} & \frac{(\gamma-1) v}{c^{2}} & -\frac{(\gamma-1)}{c^{2}}
\end{array}\right)
$$

which gives rise to the corresponding variational form of the inflow boundary conditions

$$
\delta \bar{Q}_{\beta} \Rightarrow\left(1, u, v, \frac{\mathbf{u} \cdot \mathbf{u}}{2}\right)^{T} \delta \bar{Q}_{1}
$$

on the basis of the definition of $\mathbf{T}$, from Equation (56). The preceding expression is quite distinct from Equation (43). Even though it is also simpler, it cannot be said to be the variational counterpart to fixed values of $\theta, P_{o}$ and $T_{o}$, as is the case of the latter. It must then be associated with a different set of boundary conditions.

On the other hand, the adjoint boundary operators are also given in characteristic form by [31]

$$
\mathbf{T}_{c}^{*}=\left(\begin{array}{cccc}
0 & \lambda_{2} & 0 & 0 \\
0 & 0 & \lambda_{3} & 0 \\
0 & 0 & 0 & \lambda_{4} \\
\lambda_{1} & 0 & 0 & 0
\end{array}\right)\left\{\begin{array}{l}
\mathbf{C}_{c}^{*}=\left(\begin{array}{cccc}
0 & -\lambda_{2} & 0 & 0 \\
0 & 0 & -\lambda_{3} & 0 \\
0 & 0 & 0 & -\lambda_{4}
\end{array}\right) \\
\mathbf{B}_{c}^{*}=\left(\begin{array}{cccc}
\lambda_{1} & 0 & 0 & 0
\end{array}\right)
\end{array}\right.
$$

Whence, the inverse similarity transformation yields the matrix

$$
\mathbf{T}^{*}=\left(\begin{array}{cccc}
0 & k_{y} \rho \mathbf{k} \cdot \mathbf{u} & -k_{x} \rho \mathbf{k} \cdot \mathbf{u} & \rho\left(k_{y} u-k_{x} v\right) \mathbf{k} \cdot \mathbf{u} \\
\frac{\rho \mathbf{k} \cdot \mathbf{u}}{2 c} & \frac{\rho\left(c k_{x}+u\right) \mathbf{k} \cdot \mathbf{u}}{2 c} & \frac{\rho\left(c k_{y}+v\right) \mathbf{k} \cdot \mathbf{u}}{2 c} & \frac{\rho \mathbf{k} \cdot \mathbf{u}}{2}\left(\frac{c}{(\gamma-1)}+\frac{\mathbf{u} \cdot \mathbf{u}}{2 c}+\mathbf{k} \cdot \mathbf{u}\right) \\
\frac{\rho(\mathbf{k} \cdot \mathbf{u}-c)}{2 c} & \frac{\rho\left(u-c k_{x}\right)(\mathbf{k} \cdot \mathbf{u}-c)}{2 c} & \frac{\rho\left(v-c k_{y}\right)(\mathbf{k} \cdot \mathbf{u}-c)}{2 c} & \frac{\rho(\mathbf{k} \cdot \mathbf{u}-c)}{2}\left(\frac{c}{(\gamma-1)}+\frac{\mathbf{u} \cdot \mathbf{u}}{2 c}-\mathbf{k} \cdot \mathbf{u}\right) \\
c+\mathbf{k} \cdot \mathbf{u} & u(\mathbf{k} \cdot \mathbf{u}+c) & v(\mathbf{k} \cdot \mathbf{u}+c) & \frac{(\mathbf{k} \cdot \mathbf{u}+c) \mathbf{u} \cdot \mathbf{u}}{2}
\end{array}\right)
$$

and the definition of $\mathbf{T}^{*}$, in Equation (56), leads to the following adjoint boundary condition

$$
\psi_{1}+u \psi_{2}+v \psi_{3}+\frac{\mathbf{u} \cdot \mathbf{u}}{2} \psi_{4}=0
$$

The preceding expression is, again, quite different from Equations (45)-(46), and it is much simpler, indeed. However, it is strictly associated with the linearized (60) and its corresponding flow boundary condition. Hence, it also differs from the former equations, in that it is not connected with the physical conditions from (42)-(43). 


\subsection{Outflow}

At a subsonic outflow boundary, the boundary operators of the primal problem are given, in terms of characteristic variables, by [31]

$$
\left.\begin{array}{rl}
\mathbf{B}_{c} & =\left(\begin{array}{llll}
0 & 0 & 0 & 1
\end{array}\right) \\
\mathbf{C}_{c} & =\left(\begin{array}{llll}
1 & 0 & 0 & 0 \\
0 & 1 & 0 & 0 \\
0 & 0 & 1 & 0
\end{array}\right)
\end{array}\right\} \mathbf{T}_{c}=\left(\begin{array}{llll}
0 & 0 & 0 & 1 \\
1 & 0 & 0 & 0 \\
0 & 1 & 0 & 0 \\
0 & 0 & 1 & 0
\end{array}\right)
$$

and the inverse similarity transformation yields the matrix operator

$$
\mathbf{T}=\left(\begin{array}{cccc}
\frac{(\gamma-1) \mathbf{u} \cdot \mathbf{u}+2 c \mathbf{k} \cdot \mathbf{u}}{2 c \rho} & -\frac{(\gamma-1) u+k_{x} c}{c \rho} & -\frac{(\gamma-1) v+k_{y} c}{c \rho} & \frac{(\gamma-1)}{c \rho} \\
\frac{2 c^{2}-(\gamma-1) \mathbf{u} \cdot \mathbf{u}}{2 c^{2}} & \frac{(\gamma-1) u}{c^{2}} & \frac{(\gamma-1) v}{c^{2}} & -\frac{(\gamma-1)}{c^{2}} \\
\frac{\left(k_{x} v-k_{y} u\right)}{\rho} & \frac{k_{y}}{\rho} & -\frac{k_{x}}{\rho} & 0 \\
\frac{(\gamma-1) \mathbf{u} \cdot \mathbf{u}-2 c \mathbf{k} \cdot \mathbf{u}}{2 c \rho} & \frac{k_{x} c-(\gamma-1) u}{c \rho} & \frac{k_{y} c-(\gamma-1) v}{c \rho} & \frac{(\gamma-1)}{c \rho}
\end{array}\right)
$$

which, in turn, leads to a single outflow boundary condition

$$
\delta \bar{Q}_{4}=-\left(\frac{c \mathbf{k} \cdot \mathbf{u}}{(\gamma-1)}+\frac{\mathbf{u} \cdot \mathbf{u}}{2}\right) \delta \bar{Q}_{1}+\left(\frac{c k_{x}}{(\gamma-1)}+u\right) \delta \bar{Q}_{2}+\left(\frac{c k_{y}}{(\gamma-1)}+v\right) \delta \bar{Q}_{3}
$$

Surprisingly, the preceding expression is not entirely dissimilar to Equation (49), although it does have extra terms for its coefficients. In any case, the same remark that was made regarding (60) is in order here. That is, owing to its differences, Equation (66) must be associated with flow boundary conditions other than the fixed pressure from Equation (48).

The adjoint outflow boundary conditions, in turn, are given in characteristic form by [31]

$$
\mathbf{T}_{c}^{*}=\left(\begin{array}{cccc}
0 & 0 & 0 & \lambda_{4} \\
\lambda_{1} & 0 & 0 & 0 \\
0 & \lambda_{2} & 0 & 0 \\
0 & 0 & \lambda_{3} & 0
\end{array}\right)\left\{\begin{array}{l}
\mathbf{B}_{c}^{*}=\left(\begin{array}{cccc}
\lambda_{1} & 0 & 0 & 0 \\
0 & \lambda_{2} & 0 & 0 \\
0 & 0 & \lambda_{3} & 0
\end{array}\right) \\
\mathbf{C}_{c}^{*}=\left(\begin{array}{cccc}
0 & 0 & 0 & -\lambda_{4}
\end{array}\right)
\end{array}\right.
$$

which leads to the corresponding operator $\mathbf{T}^{*}$,

$$
\mathbf{T}^{*}=\left(\begin{array}{cccc}
\frac{\rho(\mathbf{k} \cdot \mathbf{u}-c)}{2 c} & \frac{\rho\left(u-c k_{x}\right)(\mathbf{k} \cdot \mathbf{u}-c)}{2 c} & \frac{\rho\left(v-c k_{y}\right)(\mathbf{k} \cdot \mathbf{u}-c)}{2 c} & \frac{\rho(\mathbf{k} \cdot \mathbf{u}-c)}{2}\left(\frac{c}{(\gamma-1)}+\frac{\mathbf{u} \cdot \mathbf{u}}{2 c}-\mathbf{k} \cdot \mathbf{u}\right) \\
c+\mathbf{k} \cdot \mathbf{u} & u(c+\mathbf{k} \cdot \mathbf{u}) & v(c+\mathbf{k} \cdot \mathbf{u}) & \frac{1}{2} \mathbf{u} \cdot \mathbf{u}(c+\mathbf{k} \cdot \mathbf{u}) \\
0 & k_{y} \rho \mathbf{k} \cdot \mathbf{u} & -k_{x} \rho \mathbf{k} \cdot \mathbf{u} & \rho\left(k_{y} u-k_{x} v\right) \mathbf{k} \cdot \mathbf{u} \\
\frac{\rho \mathbf{k} \cdot \mathbf{u}}{2 c} & \frac{\rho\left(c k_{x}+u\right) \mathbf{k} \cdot \mathbf{u}}{2 c} & \frac{\rho\left(c k_{y}+v\right) \mathbf{k} \cdot \mathbf{u}}{2 c} & \frac{\rho \mathbf{k} \cdot \mathbf{u}}{2}\left(\frac{c}{(\gamma-1)}+\frac{\mathbf{u} \cdot \mathbf{u}}{2 c}+\mathbf{k} \cdot \mathbf{u}\right)
\end{array}\right)
$$

and the adjoint boundary conditions:

$$
\left\{\begin{array}{l}
\psi_{1}=\left(\frac{c \mathbf{k} \cdot \mathbf{u}}{(\gamma-1)}+\frac{\mathbf{u} \cdot \mathbf{u}}{2}\right) \psi_{4} \\
\psi_{2}=-\left(\frac{c k_{x}}{(\gamma-1)}+u\right) \psi_{4} \\
\psi_{3}=-\left(\frac{c k_{y}}{(\gamma-1)}+v\right) \psi_{4}
\end{array}\right.
$$

As expected, the preceding set differs considerably from Equation (51). Besides, it is strictly associated with its linearized counterpart (66) and the corresponding flow boundary condition. Hence, it is not connected with Equation (48). 


\subsection{Adjoint gradient}

The original expression for the adjoint gradient in reduced form comes from Equation (19), and it amounts to the last two surface integrals,

$$
\delta I=\int_{B_{w}} \Psi_{\alpha}\left[\delta\left(J \beta_{i^{\prime}}^{2}\right) f_{\alpha}^{i^{\prime}}+C_{\alpha \beta}^{2} \delta Q_{\beta}^{*}\right] n_{2} \mathrm{~d} \xi+\int_{B_{w}}\left\{g \delta\left|\frac{\mathrm{d} S^{\prime}}{\mathrm{d} \xi}\right|+p\left[\Psi_{\left(i^{\prime}+1\right)} \delta\left(J \beta_{i^{\prime}}^{2}\right) n_{2}\right]\right\} \mathrm{d} \xi
$$

The covariant component $n_{2}$ of the normal vector in transformed space is given in Equation (34), whereas the metric variation terms can be easily derived from Equation (35), with the aid of the aforementioned inversion relations $\eta_{, x}=-y_{, \xi} / J$ and $\eta_{, y}=x_{, \xi} / J$, and on recalling that $g_{11}=x_{, \xi}^{2}+y_{, \xi}^{2}$,

$$
\begin{gathered}
\delta\left(J \beta_{i^{\prime}}^{2}\right) n_{2}=\frac{J}{\sqrt{g_{11}}}\left\{\delta\left(J \eta_{, x}\right), \delta\left(J \eta_{, y}\right)\right\}=\frac{J}{\sqrt{g_{11}}}\left\{-\delta\left(y_{, \xi}\right), \delta\left(x_{, \xi}\right)\right\} \\
\delta\left|\frac{\mathrm{d} S^{\prime}}{\mathrm{d} \xi}\right|=\delta\left(\sqrt{g_{11}}\right)=\frac{x_{, \xi} \delta\left(x_{, \xi}\right)+y_{, \xi} \delta\left(y_{, \xi}\right)}{\sqrt{g_{11}}}
\end{gathered}
$$

From Equation (35), one can also obtain the relation $\mathrm{d} \xi=\mathrm{d} S^{\prime} / \sqrt{g_{11}}$. Then, on substituting these results for their counterparts in Equation (70), it yields the final form of the sensitivity gradient integral:

$$
\begin{aligned}
\delta I= & \int_{B_{w}}\left[\left(\psi_{\alpha} f_{\alpha}^{2^{\prime}}+\psi_{3} p+g x_{, \xi}\right) \delta\left(x_{, \xi}\right)-\left(\psi_{\alpha} f_{\alpha}^{1^{\prime}}+\psi_{2} p-g y_{, \xi}\right) \delta\left(y_{, \xi}\right)\right] \frac{\mathrm{d} S^{\prime}}{g_{11}}+ \\
& +\int_{B_{w}} \psi_{\alpha}\left(x_{, \xi} A_{\alpha \beta}^{2^{\prime}}-y_{, \xi} A_{\alpha \beta}^{1^{\prime}}\right) \delta Q_{\beta}^{*} \frac{\mathrm{d} S^{\prime}}{g_{11}}
\end{aligned}
$$

All of the metric terms in Equation (73) can be evaluated on the contour of the body solely on the basis of its parametric representation (32). Therefore, it proves the point that was made at the end of Section 3.1 to the effect that the adjoint and the optimization problems can both be solved in the physical space entirely. The mapping has fulfilled its objective in the derivation, making it simpler, and it may be dispensed with then. Under these conditions, one may simply make use of Cartesian coordinates and unstructured meshes, thus affording greater flexibility with regard to geometries. This is the approach that has been adopted in the reminder of the paper.

\section{RESULTS}

In order to validate the previous treatment of adjoint boundary conditions, it would be instructive to pursue a two-steps procedure. First, the idea is to check whether it leads to correct results in a complete application. It may well be an inverse design application, where the target geometry is known beforehand, as it was carried out in a previous paper [38]. Then, one ought to compare adjoint solutions that this treatment yields to those that are obtained under homogeneous boundary conditions at the far field. An usual definition of objective functional for inverse design applications is given by the mean square error of the actual pressure distribution $p$ with respect to a target distribution $p_{d}$,

$$
I=\frac{1}{2} \int_{B_{w}}\left(p-p_{d}\right)^{2} \mathrm{~d} s
$$

The target pressure distribution $p_{d}$ in the validation test has been obtained from the flow solution around a known geometry on making use of the same flow solver and geometry representation. The procedure ensures that not only is the target realizable but it is also attainable within the current framework. 
Flow and adjoint solutions alike were computed on unstructured meshes with triangular elements. The far-field boundary is placed over 50 chord lengths away from the airfoil surface. All geometries have been represented by the class-shape function transformation parameterization $[47,48]$. Numerical simulations for the adjoint and flow equations have been run with a cellcentered finite volume method by using the second-order five-step Runge-Kutta time-stepping scheme [49].

For simplicity, the steepest descent method was adopted as the optimization procedure. The inverse design loop consists of the following sequence: mesh generation for a given geometry, flow simulation, adjoint solution, evaluation of sensitivity gradient and gradient-based changes to geometry. The cycles are repeated until a local extremum of (74) is reached within a prescribed accuracy level.

A test case is presented next, where the initial geometry is a RAE 2822 at $M_{\infty}=0.75$ and an angle of attack of $\alpha=0^{\circ}$. The target $p_{d}$ corresponds to that of a NACA 0012 under the same flow conditions. Figure 1 shows the initial and final geometries with corresponding pressure distributions.

As the previous results show, the characteristics-based boundary conditions work properly, for the target geometry has been successfully recovered in the process: the differences between them remain below $3.5 \times 10^{-3}$. As was discussed previously, the sensitivity gradient computation here makes use of the reduced expression from Equation (73). Therefore, it only requires the adjoint solution on the airfoil contour. Figures 2 and 3 depict these solutions, as they appear at the end of the first cycle, for the upper and lower sides of the airfoil, respectively. The right side of each figure shows characteristics-based results, whereas the left side depicts results from the homogeneous boundary conditions in each case.
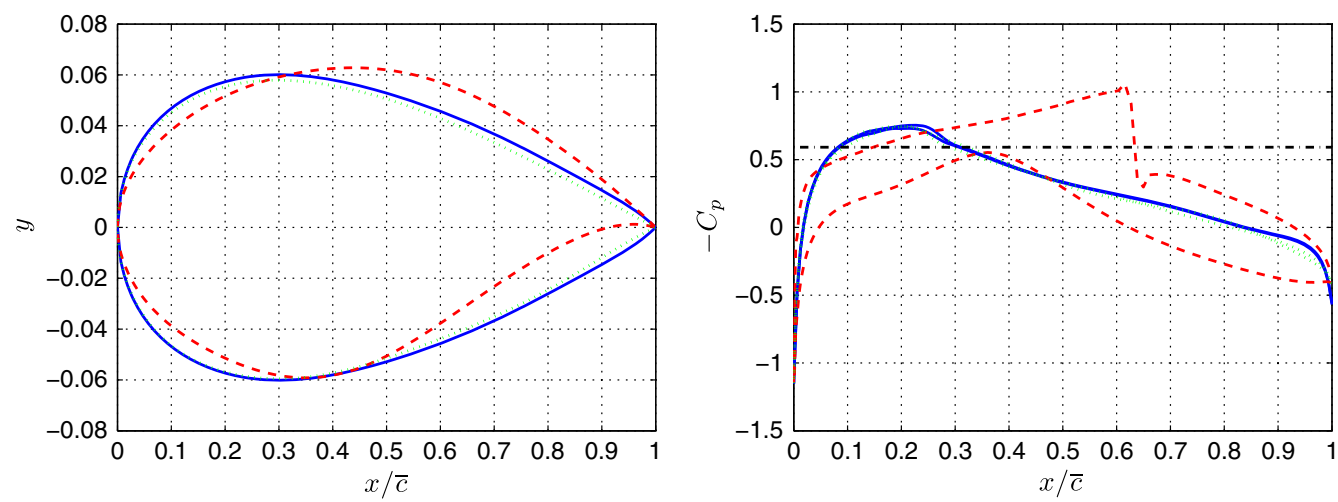

Figure 1. Mach 0.75 and $\alpha=0^{\circ}$. Left, geometries. Right, $C_{p}$. Red dash lines, original curves; green dotted lines, final results; blue solid lines, target profiles; black dash-dot, $C_{p}^{*}$.
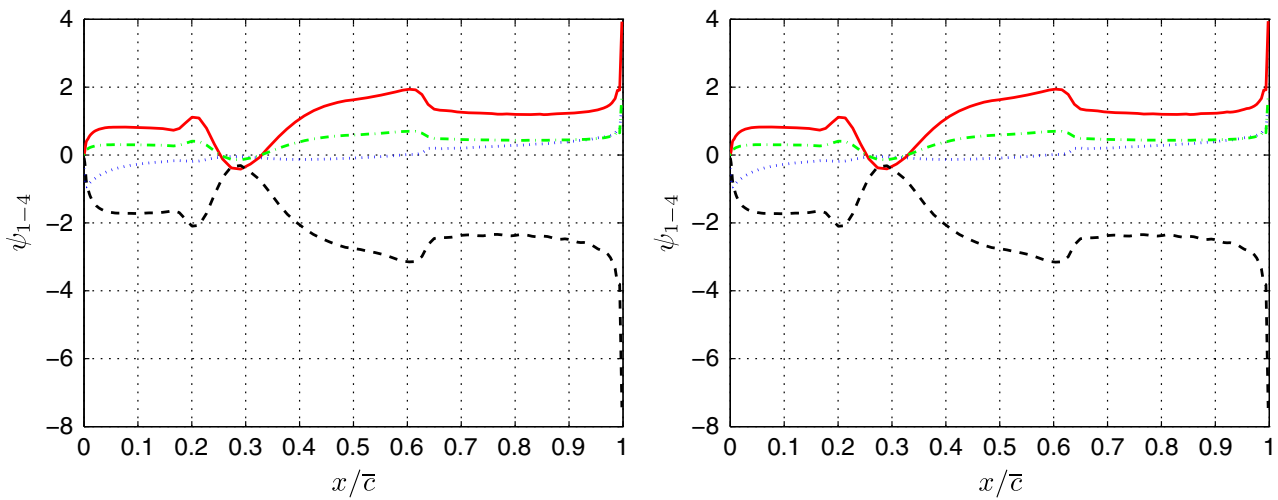

Figure 2. Airfoil upper side adjoint solution. Left, homogeneous boundary conditions. Right, characteristics-based boundary conditions. Red solid line, $\psi_{1}$; black dashed line, $\psi_{2}$; blue dotted line, $\psi_{3}$; green dash-dot, $\psi_{4}$. 

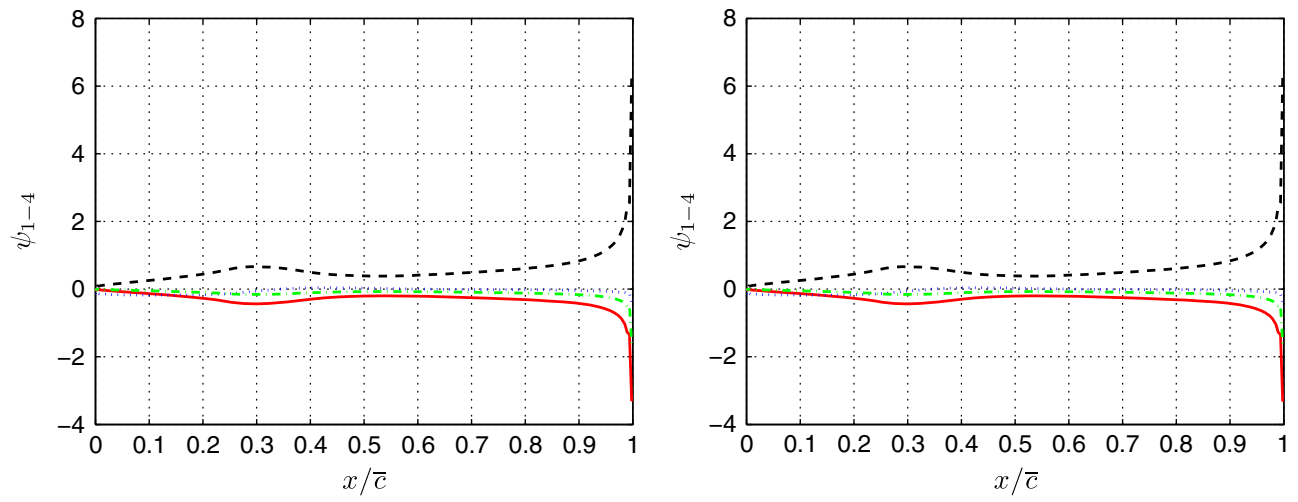

Figure 3. Airfoil lower side adjoint solution. Left, homogeneous boundary conditions. Right, characteristicsbased boundary conditions. Red solid line, $\psi_{1}$; black dashed line, $\psi_{2}$; blue dotted line, $\psi_{3}$; green dash-dot, $\psi_{4}$.

The solutions have essentially the same character over the whole airfoil contour. They even share the same discontinuity at the trailing edge. However, there is a small, yet significant, difference between them. In all points other than in the immediate neighborhood of the singularity, the difference remains below $8 \times 10^{-3}$. That value is higher than the maximum residue of the adjoint solutions $\left(7 \times 10^{-5}\right)$, hence its significance. On the other hand, that value is apparently too small to cause any noticeable differences between the two approaches in the estimates of the sensitivity gradient. In any case, the evidence suggests that further tests be devised to ascertain the influence the distance from the far-field boundary has on the adjoint solution at the contour.

Quite a different outcome is obtained for internal flows, where one does not usually have the freedom to choose the position of permeable boundaries at will. In most cases, the proximity of those boundaries makes the full characteristics approach a necessity. In order to illustrate the problem, we have computed adjoint solutions for an internal flow case by imposing homogenous and full characteristics conditions on the inflow and outflow boundaries. The same objective functional (74) was used, but the sole purpose was to solve the adjoint equations. After all, the reduced gradient form would not suit this case.

The set-up for the test is presented in Figure 4. The left side depicts initial and target geometries, while the respective $C_{p}$ distributions are shown on the right side- the latter are based on the nozzle length. Figure 5 presents the adjoint solutions at the nozzle surface. The results for homogeneous boundary conditions are on the left side, whereas those on the right side represent the full characteristics approach.
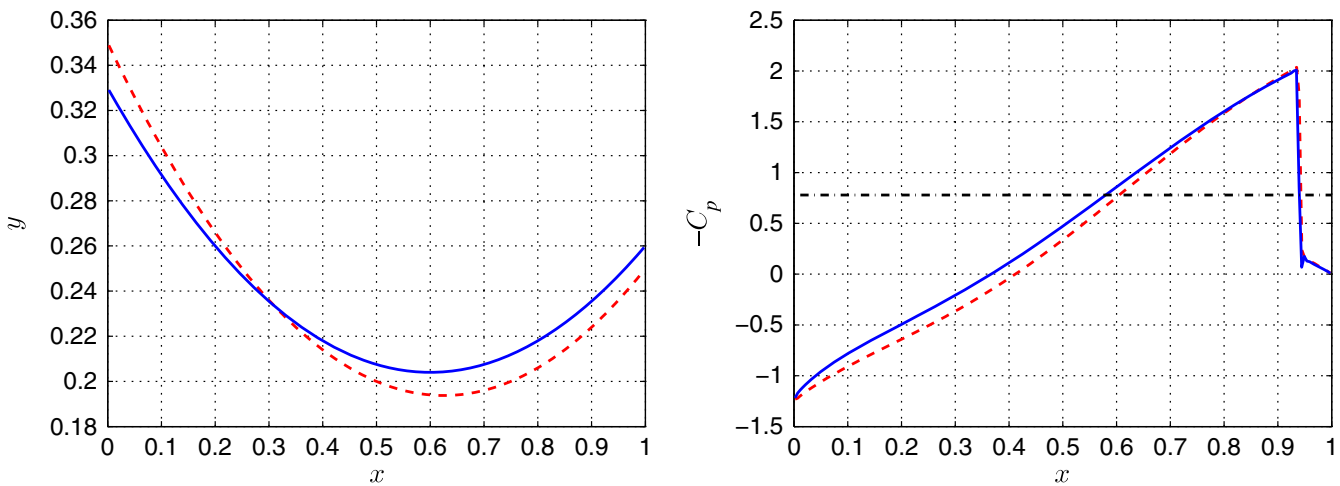

Figure 4. Mach 0.7. Left, geometries. Right, $C_{p}$. Red dashed line, original curves; blue solid line, target profiles; black dash-dot, $C_{p}^{*}$. 

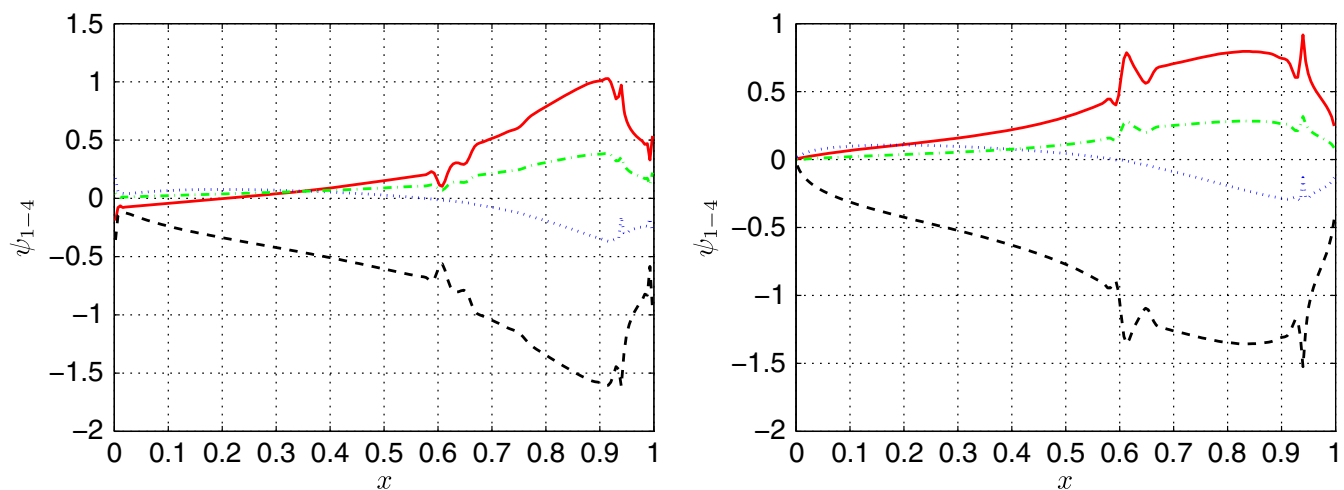

Figure 5. Left, homogeneous boundary conditions. Right, characteristics-based boundary conditions. Red solid line, $\psi_{1}$; black dashed line, $\psi_{2}$; blue dotted line, $\psi_{3}$; green dash-dot, $\psi_{4}$.

As can be seen, the differences between the solutions are quite significant, even in terms of general character. Moreover, the homogenous conditions have led to discontinuities at the boundaries, which could only be negotiated by the solver at the cost of a substantial increase in artificial dissipation.

\section{CONCLUSIONS}

The primary purpose of this work has been to investigate the adjoint boundary problem on the basis of its similarity to the corresponding flow problem. The idea was to make use of that similarity and of the particular form of the bilinear concomitant to ensure the well-posedness of the adjoint equations. To that end, two aspects of the problem have been considered at length:

First, both Euler and their adjoint equations are hyperbolic, and their characteristic velocities have the same magnitudes but opposite signs. These properties lend the primal and dual Riemann problems a complementary character, which has been thoroughly investigated by Giles and Pierce in their seminal work on the topic. In fact, that complementarity is what enables one to make the adjoint equations well posed while also eliminating all physical variations from the augmented variational problem.

The second aspect specifically concerns the bilinear concomitant. It is well known that its particular form can be seen as an inner product between physical variations and adjoint variables, which is computed at the boundaries of the flow domain. The proposed approach combines this interpretation with the idea that flow boundary conditions constrain physical variations to a locus of realizability in state space. Hence, the need to drive the bilinear concomitant to zero is tantamount to requiring that the vector of adjoint variables be orthogonal to all realizable variations. The rationale behind the procedure is analogous to the principle of virtual work in that, as a constraint force, the adjoint vector must be normal to all feasible displacements of the system. The expressions this procedure yields have been termed compatibility conditions.

The algebra has been shown to yield a balance between the compatibility conditions and the corresponding number of domain incoming characteristics. Therefore, one is able to put together a complete set of adjoint Riemann equations for each permeable boundary. The end result is a set of boundary conditions that is entirely similar to those that render the Euler equations well posed. The solid wall is the sole exception, because it entails only one domain incoming characteristic for each problem, flow and adjoint. As Giles and Pierce point out, the flux Jacobian matrix associated with the normal direction to the wall becomes singular. Hence, the adjoint Riemann set becomes indeterminate, and one must rely on the numerical extrapolation schemes.

A series of validation tests was devised to verify the proposed approach. For external flows, the results show good agreement with the conventional homogeneous far-field conditions for the adjoint problem. Nonetheless, there are small, yet apparently significant, differences between the two. Further tests are required to ascertain the influence the position of the far-field boundary 
exerts on the overall solution. As for internal flows, test results have shown the full-characteristics conditions to yield smooth solutions in the neighborhood of permeable boundaries, as opposed to the discontinuities that result from the homogeneous ones. That piece of evidence, along with an analogy with the flow physics, seems to indicate that the latter treatment of the boundary problem is inappropriate.

Given its central role in the theory, it should be stressed that the bilinear concomitant effectively connects flow and adjoint boundary problems. As the name implies, it consists of bilinear products between physical variations and adjoint variables, where the former are constrained by flow physics. Hence, the need to drive it to zero, while preserving well-posedness, does limit the possibilities of boundary conditions one can impose on the adjoint equations. The connection also implies that there must be a precise correspondence between flow and adjoint solutions in terms of the boundary conditions they meet, for that is the only means of ensuring that the bilinear concomitant vanishes for arbitrary physical variations.

The only exception to the rule seems to be the imposition of homogeneous adjoint boundary conditions at the far field, for obvious reasons. Although they may represent an over-specification on purely mathematical grounds, they are often a perfectly valid approximation to the characteristicsbased formulation. Such appears to be the case with so many applications in external flows, where that boundary is suitably placed far enough from the body as to ensure all flow-field perturbations die away before they ever reach it.

\section{ACKNOWLEDGEMENTS}

The authors acknowledge the contribution of the NDF laboratory at the University of São Paulo in providing the required resources for the execution of this work. The first author also acknowledges the Conselho Nacional de Desenvolvimento Científico e Tecnológico (CNPq) for his PhD scholarship.

\section{REFERENCES}

1. Jameson A. Aerodynamic design via control theory. Journal of Scientific Computing 1988; 3(f3):233-260.

2. Pirroneau O. On optimal profiles in Stokes flow. Journal of Fluid Mechanics 1973; 59(1):117-128.

3. Pirroneau O. On optimal design in fluid dynamics. Journal of Fluid Mechanics 1974; 64(1):97-110.

4. Pirroneau O. Optimal Shape Design for Elliptic Systems, 1st edition. Springer-Verlag: Berlin, Heidelberg, New York, Tokyo, 1983.

5. Alonso JJ, Kroo IM, Jameson A. Advanced algorithms for design and optimization of quiet supersonic platforms. In AIAA Computational Fluid Dynamics Conference, Reno, NV, January 2002; 13. AIAA-2002-0144.

6. Cabuk H, Sung CH, Modi V. Adjoint operator approach to shape design for incompressible flows. In 3rd International Conference on Inverse Design Concepts and Optimization in Engineering Sciences (ICIDES), College Park, PA, 1991; 391-404.

7. Jameson A. Aerodynamic design via control theory. Journal of Scientific Computing 1988; 3:233-260.

8. Jameson A. Optimum aerodynamic design using CFD and control theory. AIAA 12th Computational Fluid Dynamics Conference. In AIAA 95-1729-CP: San Diego, 1995.

9. Jameson A, Martinelli L, Pierce NA. Optimum aerodynamic design using the Navier-Stokes equations. In 35th Aerospace Sciences Meeting \& Exhibit, Reno, NV, January 1997; 1-20. American Institute of Aeronautics and Astronautics, AIAA.

10. Kim S. Design optimization of high-lift configurations using a viscous adjoint-based method. PhD thesis, Stanford University, 2001.

11. Kuruvila G, Taasan S, Salas MD. Airfoil optimization by the one-shot method, optimum design methods in aerodynamics. AGARD-FDP-VKI Special Course, 1994.

12. Mohammadi B, Pirroneau O. Applied Shape Optimization for Fluids, 1st edition. Oxford University Press: New York, 2001.

13. Santos LCC. A study on aerodynamic design optimization using an adjoint method. Technical Report IB 129 - 95/12, Institut für Entwurfsaerodynamik, Braunschweig, July 1995.

14. Taasan S, Kuruvila G, Salas MD. Aerodynamic design and optimization in one shot. In 30th Aerospace Sciences Meeting and Exhibit, Reno, NV, January 1992; 16. AIAA 92-0025.

15. Cacuci DG, Weber CF, Oblow EM, Marable JH. Sensitivity theory for general systems of non-linear equations. Nuclear Science and Engineering 1980; 75:88-110.

16. Hall MCG, Cacuci D. Physical interpretation of the adjoint functions for sensitivity analysis of atmospheric models. Journal of Atmospheric Sciences October 1983; 40:2537-2546.

17. Duta MC, Giles MB, Campobasso MS. The harmonic adjoint approach to unsteady turbomachinery design. International Journal for Numerical Methods in Fluids 2002; 40:323-332. 
18. Kim H, Nakahashi K. Unstructured adjoint method for Navier-Stokes equations. JSME International Journal 2005; 48(2):202-207.

19. Kim HJ, Sasaki D, Obayashi S, Nakahashi K. Aerodynamic optimization of supersonic transport wing using unstructured adjoint method. AIAA Journal June 2001; 39(6):1011-1020.

20. Kim S, Alonso JJ, Jameson A. Multi-element high-lift configuration design optimization using viscous continuous adjoint method. Journal of Aircraft 2004; 41(5):1082-1097.

21. Nadarajah SK. The discrete adjoint approach to aerodynamic shape optimization. PhD thesis, Stanford University, 2003.

22. Qiao Z, Qin X, Yang X. Wing design by solving adjoint equations. In 40th Aerospace Sciences Meeting \& Exhibit, Reno, NV, January 2002. American Institute of Aeronautics and Astronautics, AIAA.

23. Mani K, Mavriplis D. Unsteady discrete adjoint formulation for two-dimensional flow problems with deforming meshes. AIAA Journal June 2008; 46(6):1351-1364.

24. Nadarajah SK, Jameson A. Optimum shape design for unsteady three-dimensional viscous flows using a non-linear frequency domain method. In 24th Applied Aerodynamics Conference, San Francisco, CA, June 2006; 22. American Institute of Aeronautics and Astronautics, AIAA.

25. Nadarajah SK, Jameson A. Optimum shape design for unsteady flows with time-accurate continuous and discrete adjoint methods. AIAA Journal July 2007; 45(7):1478-1491.

26. Thomas JP, Hall KC, Dowell EH. Discrete adjoint approach for modeling unsteady aerodynamic design sensitivities. AIAA Journal September 2005; 43(9):1931-1936.

27. Jameson A, Martinelli L, Pierce NA. Optimum aerodynamic design using the Navier-Stokes equations. Theoretical and Computational Fluid Dynamics 1998; 1(10):213-237.

28. Jameson A, Sriram A, Martinelli L. A continuous adjoint method for unstructured grids. In AIAA Computational Fluid Dynamics Conference, Orlando, FL, June 2003; 16. AIAA 2003-3955.

29. Jameson A, Nadarajah SK. A comparison of the continuous and discrete adjoint approach to automatic aerodynamic optimization. In AIAA 38th Aerospace Sciences Meeting and Exhibit, Reno, NV, January 2000; 20. AIAA $2000-0667$.

30. Kim S, Alonso JJ, Jameson A. A gradient accuracy study for the adjoint-based Navier-Stokes design method. AIAA 37th Aerospace Sciences Meeting and Exhibit, Reno, NV, January 1999. AIAA-99-0299.

31. Giles MB, Pierce NA. Adjoint equations in CFD: duality, boundary conditions and solution behavior. AIAA Paper 97-1850, 1997.

32. Giles MB, Pierce NA. On the properties of solutions of the adjoint Euler equations. In Numerical Methods for Fluid Dynamics VI, Baines MJ (ed.). ICFD: Oxford, UK, June 1998.

33. Giles MB, Pierce NA. Analytic adjoint solutions for the quasi-1D Euler equations. Report 00/03, Oxford University Computing Laboratory, Oxford, March 2000.

34. Giles MB, Pierce NA. Analytic adjoint solutions for the quasi-1D Euler equations. Journal of Fluid Mechanics 2001; 426:327-345.

35. Hirsch C. Numerical Computation of Internal and External Flows: Computational Methods for Inviscid and Viscous Flows, 1st edition, volume 2 of Wiley Series in Numerical Methods in Engineering. John Wiley \& Sons: NY, 1994.

36. Hayashi MT. Estudo conceitual do problema adjunto baseado nas equações de Euler para aplicações de otimização aerodinâmica. Master's thesis, Escola Politécnica da Universidade de São Paulo, Fevereiro, 2009.

37. Hayashi MT, Ceze MAB, Volpe EV. Characteristics-based boundary conditions for the Euler adjoint problem: 2-D formulation. In AIAA Applied Aerodynamics Conference, Chicago, IL, June 2010; 18. AIAA. 2010-4677.

38. Volpe EV, Santos LCC. Boundary and internal conditions for adjoint fluid flow problems - applications to quasi-1D Euler equations. Journal of Engineering Mathematics September 2009; 65(1):1-24.

39. Gelfand IM, Fomin SV. Calculus of Variations, 1st edition, Selected Russian Publications in Mathematical Sciences. Prentice-Hall, Inc.: N.J., 1963. Translated and Edited by R. A. Silverman.

40. Reuther JJ. Aerodynamic shape optimization using control theory. PhD thesis, University of California, 1996.

41. Jameson A, Kim S. Reduction of the adjoint gradient formula for aerodynamic shape optimization problems. AIAA Journal November 2003; 41(11):2114-2129.

42. Carrier GF, Pearson CE. Partial Differential Equations: Theory and Technique, 2nd edition. Academic Press: NY, 1988.

43. Hirsch C. Numerical Computation of Internal and External Flows: Fundamentals of Numerical Discretization, 1st edition, volume 1 of Wiley Series in Numerical Methods in Engineering. John Wiley \& Sons: NY, 1994.

44. Flügge W. Tensor Analysis and Continuum Mechanics, 1st edition. Springer-Verlag: N.Y., 1972.

45. Kaplan W. Advanced Calculus. Addison-Wesley Pub. Co.: NY, 1971.

46. Ceze MAB. Projeto inverso aerodinâmico utilizando o método adjunto aplicado às equações de Euler. Master's thesis, Escola Politécnica da Universidade de São Paulo, Agosto 2008.

47. Ceze MAB, Hayashi MT, Volpe EV. A study of the CST parameterization characteristics. In AIAA Applied Aerodynamics Conference, San Antonio, TX, June 2009; 12. AIAA. AIAA 2009-3767.

48. Kulfan BM, Bussoletti JE. Fundamental parametric geometry representations for aircraft component shapes. In 11th AIAA/ISSMO Multidisciplinary Analysis and Optimization Conference, Portsmouth, VA, September 2006; 45.

49. Jameson A, Schmidt W, Turkel E. Numerical solution of the Euler equations by finite volume methods using Runge-Kutta time-stepping schemes. AIAA 14th Fluid and Plasma Dynamic Conference, Palo Alto, June 1981; 15. AIAA Paper 81-1259. 